Swarthmore College

Works

\title{
The Degree Of Alignment Between Circumbinary Disks And Their Binary Hosts
}

I. Czekala

E. Chiang

S. M. Andrews

Eric L.N. Jensen

Swarthmore College, ejensen1@swarthmore.edu

G. Torres

See next page for additional authors

Follow this and additional works at: https://works.swarthmore.edu/fac-physics

Let us know how access to these works benefits you

\section{Recommended Citation}

I. Czekala, E. Chiang, S. M. Andrews, Eric L.N. Jensen, G. Torres, D. J. Wilner, K. G. Stassun, and B. Macintosh. (2019). "The Degree Of Alignment Between Circumbinary Disks And Their Binary Hosts". Astrophysical Journal. Volume 883, Issue 1. DOI: 10.3847/1538-4357/ab287b https://works.swarthmore.edu/fac-physics/370

This work is brought to you for free by Swarthmore College Libraries' Works. It has been accepted for inclusion in Physics \& Astronomy Faculty Works by an authorized administrator of Works. For more information, please contact myworks@swarthmore.edu. 
Authors

I. Czekala, E. Chiang, S. M. Andrews, Eric L.N. Jensen, G. Torres, D. J. Wilner, K. G. Stassun, and B. Macintosh 


\title{
The Degree of Alignment between Circumbinary Disks and Their Binary Hosts
}

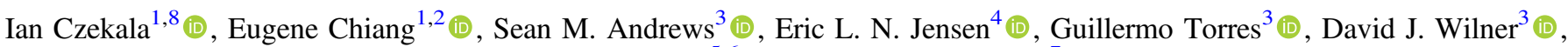 \\ Keivan G. Stassun ${ }^{5,6}$ (D), and Bruce Macintosh ${ }^{7}$ (i) \\ ${ }^{1}$ Department of Astronomy, University of California at Berkeley, Campbell Hall, CA 94720-3411, USA; iczekala@berkeley.edu \\ 2 Department of Earth and Planetary Science, University of California at Berkeley, McCone Hall, Berkeley, CA 94720-4767, USA \\ ${ }^{3}$ Center for Astrophysics, Harvard \& Smithsonian, 60 Garden Street, Cambridge, MA 02138, USA \\ ${ }^{4}$ Department of Physics and Astronomy, Swarthmore College, 500 College Avenue, Swarthmore, PA 19081, USA \\ ${ }^{5}$ Department of Physics and Astronomy, Vanderbilt University, 6301 Stevenson Center, Nashville, TN 37235, USA \\ ${ }^{6}$ Department of Physics, Fisk University, Nashville, TN 37208, USA \\ ${ }^{7}$ Kavli Institute for Particle Astrophysics and Cosmology, Stanford University, 452 Lomita Mall, Stanford, CA 94305, USA \\ Received 2019 March 25; revised 2019 June 1; accepted 2019 June 6; published 2019 September 17
}

\begin{abstract}
All four circumbinary $(\mathrm{CB})$ protoplanetary disks orbiting short-period $(P<20$ days) double-lined spectroscopic binaries (SB2s) - a group that includes UZ Tau E, for which we present new Atacama Large Millimeter/ Submillimeter Array data - exhibit sky-plane inclinations $i_{\text {disk }}$ that match, to within a few degrees, the sky-plane inclinations $i_{\star}$ of their stellar hosts. Although for these systems the true mutual inclinations $\theta$ between disk and binary cannot be directly measured because relative nodal angles are unknown, the near coincidence of $i_{\text {disk }}$ and $i_{\star}$ suggests that $\theta$ is small for these most compact of systems. We confirm this hypothesis using a hierarchical Bayesian analysis, showing that $68 \%$ of CB disks around short-period SB2s have $\theta<3{ }^{\circ} 0$. Near coplanarity of CB disks implies near coplanarity of $\mathrm{CB}$ planets discovered by Kepler, which in turn implies that the occurrence rate of close-in CB planets is similar to that around single stars. By contrast, at longer periods ranging from 30 to $10^{5}$ days (where the nodal degeneracy can be broken via, e.g., binary astrometry), CB disks exhibit a wide range of mutual inclinations, from coplanar to polar. Many of these long-period binaries are eccentric, as their component stars are too far separated to be tidally circularized. We discuss how theories of binary formation and disk-binary gravitational interactions can accommodate all these observations.
\end{abstract}

Key words: binaries: close - binaries: spectroscopic - planet-disk interactions - protoplanetary disks - stars: pre-main sequence

\section{Introduction}

A fact no less true for being so commonly stated, most solartype stars do indeed reside in binary or higher-multiplicity systems (Raghavan et al. 2010). The influence of binarity on star and planet formation has primarily been addressed from the perspective of circumstellar disks or planets perturbed by an external stellar companion (i.e., in the " $\mathrm{S}$ "-type configuration; Dvorak 1982). For example, the presence of a binary companion with semimajor axis $a<50$ au will truncate the outer radius of a circumstellar disk (Jensen et al. 1996b; Harris et al. 2012), possibly reducing the planet occurrence rate (Wang et al. 2014; Kraus et al. 2016). By comparison, at the closest binary separations $(a \lesssim 10$ au), massive disks will most likely be circumbinary (i.e., in the "P"-type configuration; Dvorak 1982; Harris et al. 2012).

Efforts to study planet formation in the circumbinary sense were given new urgency by the Kepler mission's discovery of circumbinary (CB) planets orbiting eclipsing binary (EB) stars (e.g., Doyle et al. 2011; Orosz et al. 2012; Welsh et al. 2012; Schwamb et al. 2013). Thus far, the dozen known transiting CB planets orbit binaries whose periods $P$ fall in the range of 7-40 days and are inclined relative to their host EB planes by $\theta<5^{\circ}$ (see the compilation by Winn \& Fabrycky 2015). The underlying distribution of mutual inclinations is not well constrained. Because the Kepler CB planet sample is sourced from the Kepler EB sample (which have sky-plane inclinations $i_{\star} \simeq 90^{\circ}$, i.e., the binaries are viewed nearly edge-on), the Kepler CB planet survey

\footnotetext{
${ }^{8}$ NASA Hubble Fellowship Program Sagan Fellow.
}

has poor sensitivity to planets on orbits with large mutual inclinations. Even if an inclined planet were to transit once, subsequent transits occurring every planetary orbital period would not be guaranteed because the transited star would have moved in its orbit (Martin \& Triaud 2014). Knowing the mutual inclination distribution is necessary for calculating the intrinsic occurrence rate of Kepler CB planets (Armstrong et al. 2014). Statistical and dynamical arguments suggest $\theta \lesssim 3^{\circ}$ ( $\mathrm{Li}$ et al. 2016), while the nondetections of the BEBOP radial velocity (RV) survey, which is sensitive to CB giant planets around single-lined EBs with periods $P<40$ days, can be combined with Kepler statistics to infer that $\theta \lesssim 10^{\circ}$ (Martin et al. 2019). Other detection techniques such as $\mathrm{RV}$, eclipse timing variations, microlensing, and direct imaging have also been employed to search for CB planets, though their samples are smaller than Kepler's and their survey selection functions are often more difficult to characterize (e.g., Udry et al. 2002; Bailey et al. 2014; Bennett et al. 2016; Asensio-Torres et al. 2018; De Rosa \& Kalas 2019).

Another way to get at $\mathrm{CB}$ planet inclinations, and gain broader insight into the formation of CB planets in general, is to study CB disks, either of the protoplanetary or debris variety. Spatially resolved observations of CB disks constrain their skyplane inclinations $i_{\text {disk }}$ and position angles of their ascending nodes $\Omega_{\text {disk }}$ (see Figure 1 ). ${ }^{9}$ Combining these disk parameters

\footnotetext{
9 We follow the convention of the visual binary field and throughout this paper label the ascending node as the one at which the secondary is receding from the observer (e.g., van de Kamp 1981; Torres 1995; Pourbaix 1998), whose position angle $\Omega$ is measured by the number of degrees east of north (counterclockwise on the sky). The approaching node is sometimes used as the ascending node in the exoplanet field (e.g., Murray \& Correia 2010).
} 


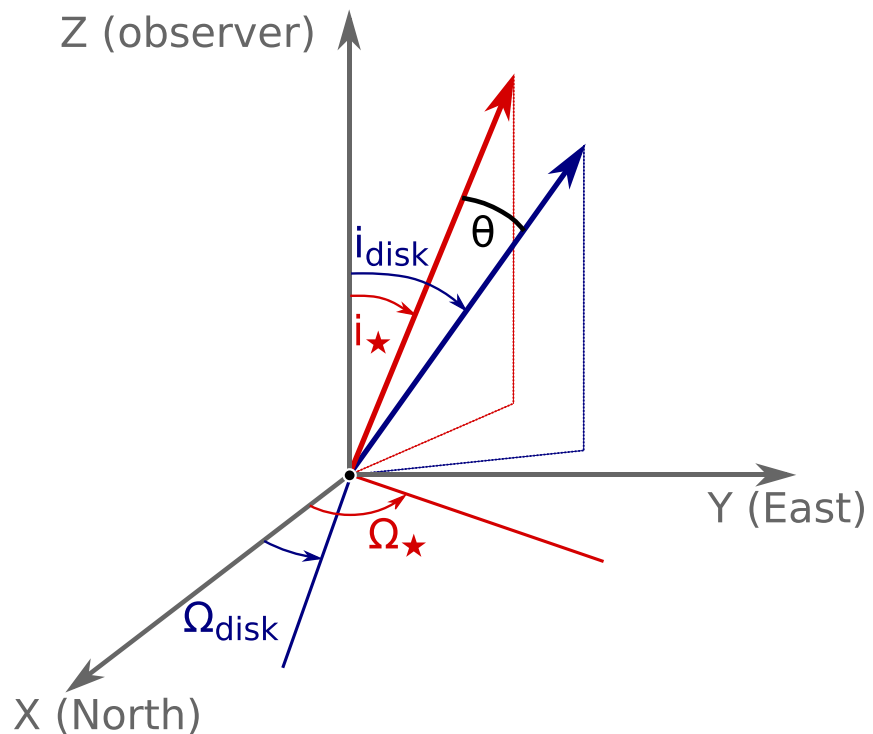

Figure 1. Orientations of a binary orbit and its circumbinary disk are denoted using their unit orbital angular momentum vectors (in red and blue, respectively). The mutual inclination $\theta$ is the angle between these two vectors. For an observer located at $Z=+\infty$, the inclination $i$ is defined as the angle between the orbit vector and the $Z$-axis. The position angle of the ascending node $\Omega$ defines the orientation of the orbit in the tangent sky plane (the $X-Y$ plane).

with measurements of the sky-plane binary inclination $i_{\star}$ and ascending node $\Omega_{\star}$ enables calculation of the mutual diskbinary inclination $\theta$ :

$$
\cos \theta=\cos i_{\text {disk }} \cos i_{\star}+\sin i_{\text {disk }} \sin i_{\star} \cos \left(\Omega_{\text {disk }}-\Omega_{\star}\right) .
$$

Joint RV and astrometric observations yield $i_{\star}$ and $\Omega_{\star}$ directly. For the shortest-period binaries that are astrometrically inaccessible, it is still possible to measure $i_{\star}$ by combining a double-lined spectroscopic RV measurement of $\left(M_{1}+M_{2}\right) \sin ^{3} i_{\star}$ with an estimate of the total stellar mass $M_{1}+M_{2}$ from the disk rotation curve. This leaves only $\Omega_{\star}$ unspecified. Unfortunately, with a uniform prior on $\Omega_{\star}$, there still remains a wide range of possible mutual inclinations, particularly for systems not viewed face-on.

The situation for short-period systems, however, is not necessarily as uncertain as the above considerations suggest. Suppose that the intrinsic distribution of disk-binary inclinations were broad. In that case, it would be surprising to measure $i_{\text {disk }}$ and $i_{\star}$ in a given system and find these two angles to be nearly identical. The more often we observed this nearequality, the more surprised we would be. And yet that is precisely the hand we have been dealt: of the four known $\mathrm{CB}$ protoplanetary disks orbiting $P<20$ day double-lined spectroscopic binaries (SB2s), all have $i_{\text {disk }} \simeq i_{\star}$ to within a few degrees. This measurement outcome leads us to suspect that the deck is stacked: that we do not live in a universe where mutual inclinations are random, but one in which they are preferentially small, at least for these most compact of systems.

A large portion of this paper is devoted to proving, in a statistically rigorous manner, that most circumbinary disks orbiting short-period binaries are nearly coplanar (see also Prato et al. 2002; Jensen et al. 2007; Kennedy et al. 2012a). We will implement a hierarchical Bayesian analysis that leverages the (incomplete) data we have for CB disks orbiting shortperiod SB2s to infer, in full, the intrinsic distribution of mutual inclinations $\theta$ from which they are drawn. We will supplement this analysis by compiling a database of $\mathrm{CB}$ disks orbiting longer-period binaries, to search for possible trends between disk-binary mutual inclination and other system parameters such as binary period and binary eccentricity.

We begin our study by using new Atacama Large Millimeter/Submillimeter Array (ALMA) data to update the parameters of one of the four $\mathrm{CB}$ disks orbiting short-period SB2s, UZ Tau E. In Section 2, we present ${ }^{12} \mathrm{CO},{ }^{13} \mathrm{CO}$, and $\mathrm{C}^{18} \mathrm{O} J=2-1$ data for UZ Tau E, derive a new dynamical mass for the central binary, and measure $i_{\star}$ (but not $\Omega_{\star}$ ). In Section 3 we assemble a collection of circumbinary protoplanetary and debris disk systems from the literature and calculate their mutual inclinations-in some cases only in a statistical sense. There we implement a hierarchical Bayesian model to infer the underlying mutual inclinations of the subsample of CB disks around SB2s. In Section 4 we discuss our observations in the context of theories of binary formation and disk-binary gravitational interactions and make connections to the population of Kepler CB planets. We conclude in Section 5 .

\section{UZ Tau Data and Analysis}

We review what is known about UZ Tau, which actually comprises two binaries, in Section 2.1. We describe our new ALMA data and how we reduced them in Section 2.2. By forward-modeling the molecular line emission, we calculate disk and binary inclination parameters for UZ Tau E in Section 2.3.

\subsection{Background Data on UZ Tau}

The UZ Tau system consists of UZ Tau E, which is a doublelined spectroscopic binary consisting of Ea and $\mathrm{Eb}(P=19.131$ days, $e=0.33, q \equiv M_{2} / M_{1}=0.30$; Jensen et al. 1996a, 2007; Mathieu et al. 1996; Prato et al. 2002), and UZ Tau W, which is a visual binary consisting of $\mathrm{Wa}$ and $\mathrm{Wb}$, separated by 0 !" 3 . The $\mathrm{E}$ and W binaries are separated by 3."6 (Correia et al. 2006). The $\mathrm{Ea}$ and $\mathrm{Eb}$ stars have spectral types M1 and M4, respectively (Prato et al. 2002), and the $\mathrm{Wa}$ and $\mathrm{Wb}$ stars are both $\mathrm{M} 2$ spectral type (Correia et al. 2006). A Gaia DR2 parallax exists for UZ Tau E of $\pi=7.62 \pm 0.10$ mas (including a 0.02 mas systematic term; Lindegren et al. 2018) or $131.2 \pm 1.7 \mathrm{pc}$ (Bailer-Jones et al. 2018; Gaia Collaboration et al. 2018).

Simon et al. (2000) observed the UZ Tau E CB disk using ${ }^{12} \mathrm{CO} J=2-1$ observations from the IRAM interferometer. They forward-modeled the disk rotation to derive a total stellar mass of $M_{\mathrm{Ea}}+M_{\mathrm{Eb}}=1.31 \pm 0.08 M_{\odot}$ (assuming $d=140 \mathrm{pc}$ ). However, some uncertainty remains in this result owing to severe ${ }^{12} \mathrm{CO}$ contamination from the molecular cloud and the modest spatial and spectral resolution of the observations (1". 1 and $1.2 \mathrm{~km} \mathrm{~s}^{-1}$, respectively). Nevertheless, Prato et al. (2002) combined this disk-based measurement with the double-lined $\mathrm{RV}$ solution to find $\left|i_{\text {disk }}-i_{\star}\right|<5^{\circ}$. We obtained new highresolution ALMA observations of the UZ Tau system in the ${ }^{12} \mathrm{CO},{ }^{13} \mathrm{CO}$, and $\mathrm{C}^{18} \mathrm{O} J=2-1$ transitions in order to derive new dynamical mass measurements of UZ Tau E and remove the lingering uncertainties.

Recent submillimeter-, millimeter-, and centimeter-wave continuum observations of UZ Tau have also clarified the distribution of dust in the quadruple system. Tripathi et al. (2018) studied UZ Tau across a broad range of radio and millimeter wavelengths and spatially resolved individual 

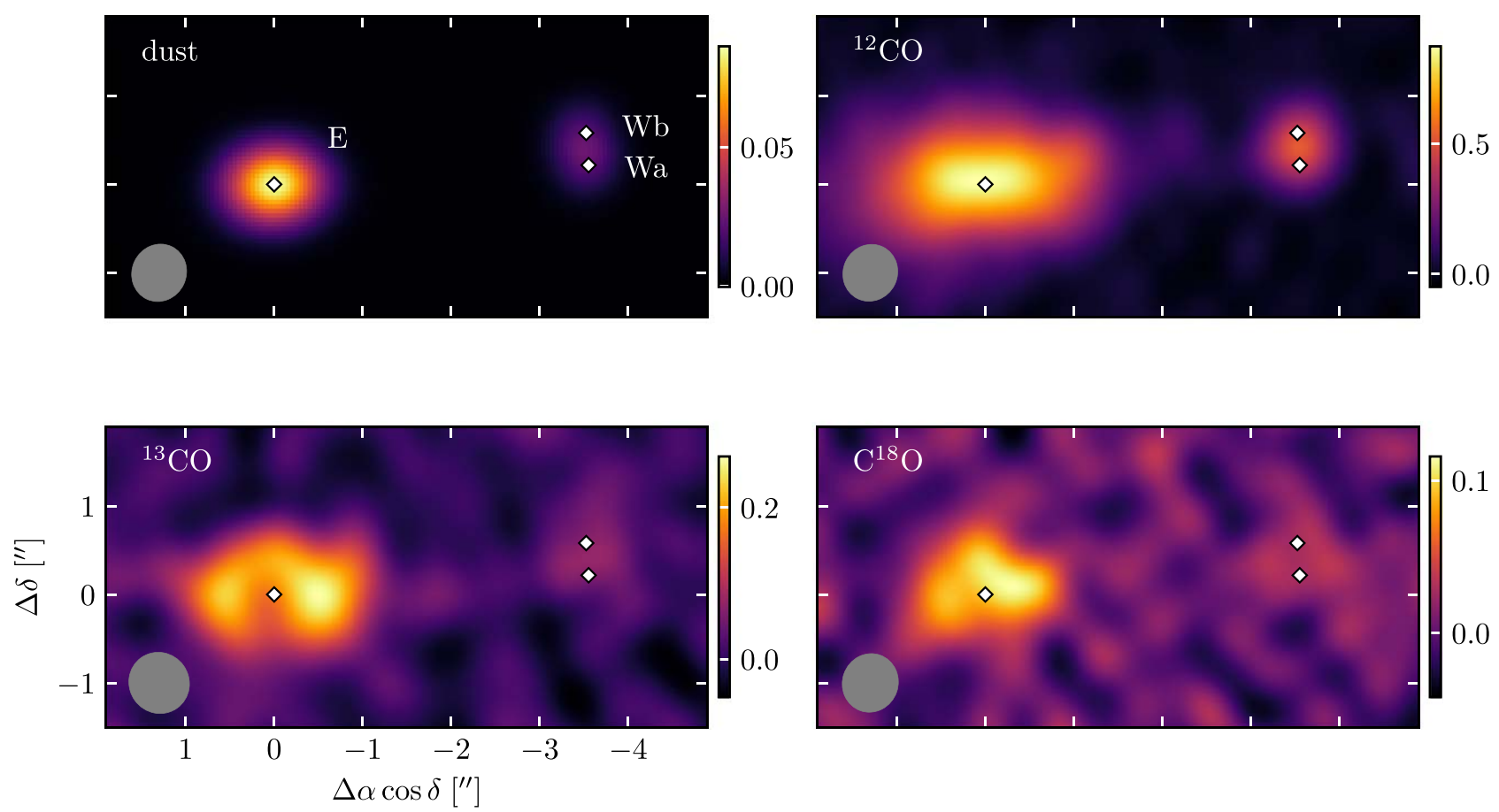

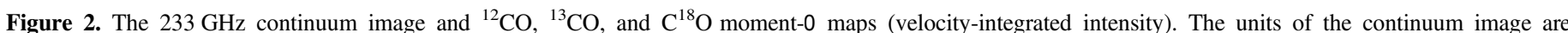

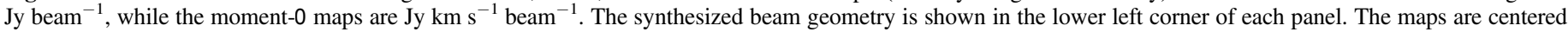

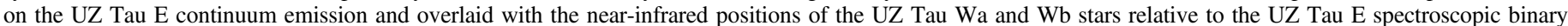
(Correia et al. 2006).

circumstellar disks around $\mathrm{Wa}$ and $\mathrm{Wb}$ for the first time. Long et al. (2018) targeted UZ Tau E with high-resolution ( 0 ". 12) ALMA observations of the dust continuum. They found disk substructures in the form of a small inner cavity $(r<15 \mathrm{au})$ and two low-contrast axisymmetric emission rings at $\sim 20$ and $\sim 75 \mathrm{au}$. They also measured the disk inclination (relative to the sky plane) to be $i_{\text {disk }}=56^{\circ} .15 \pm 1.50$ and the position angle of the ascending node to be $\Omega_{\text {disk }}=90^{\circ} .39 \pm 1.50$ (systematic uncertainties included).

\subsection{New ALMA Observations of UZ Tau}

We observed the UZ Tau system with ALMA on 2016 June 21 under project code 2015.1.00690.S. The array was composed of 36 antennas, and the baselines ranged from 15 to $704 \mathrm{~m}$. This configuration of the array yielded a maximum recoverable scale of 10 ". 7 . The total on-source time was 22 minutes. Our band 6 observations targeted the dust continuum with a $1.875 \mathrm{GHz}$ wide spectral window centered at $233.000 \mathrm{GHz}$, and we placed three spectral windows on the ${ }^{12} \mathrm{CO},{ }^{13} \mathrm{CO}$, and $\mathrm{C}^{18} \mathrm{O} J=2-1$ molecular transitions with channel spacings of $30.5,30.5$, and $61.0 \mathrm{kHz}$, respectively. The Hanning smoothing window applied by the ALMA correlator slightly correlates the information content of adjacent channels, suppressing Gibbs ringing but effectively reducing the resolution by an additional factor of $\approx 2$, resulting in effective velocity resolutions of 79,83 , and $167 \mathrm{~m} \mathrm{~s}^{-1}$, respectively.

To create a set of continuum-only visibilities, we used the CASA task plotms (McMullin et al. 2007) to identify channels with strong line emission, excised these, and averaged the remaining channels to create a measurement set with a total of $2.344 \mathrm{GHz}$ continuum bandwidth. We used the CASA task clean to image the continuum and placed masks around the visible sources UZ Tau E and W. We used this CLEAN model to perform three rounds of phase-only self-calibration and one round of amplitude and phase self-calibration via the gaincal task. After applying the solution to the measurement set, the final rms in the continuum channel was reduced from 450 to $70 \mu \mathrm{Jy}_{\text {beam }}{ }^{-1}$.

We then applied the self-calibration solution to the full data set, including the spectral windows containing line emission. The continuum was estimated in the visibility plane and subtracted from the data set using the CASA task uvcontsub. An initial round of imaging with tclean revealed that emission from all isotopologues was clearly detected, with the brightest, ${ }^{12} \mathrm{CO}$, spanning a line width of nearly $11 \mathrm{~km} \mathrm{~s}^{-1}$. We used the task mstransform to correct the spectral line channels to the reference frame of the kinematic local standard of rest (LSRK) and binned all line spectral windows into matching channels $334 \mathrm{~m} \mathrm{~s}^{-1}$ wide. We chose this channel width, representing binning factors of $\sim 8, \sim 8$, and 4 for the ${ }^{12} \mathrm{CO},{ }^{13} \mathrm{CO}$, and $\mathrm{C}^{18} \mathrm{O}$ spectral windows, respectively, because this produces effectively independent channels for all transitions on the same velocity scale and is a sensible compromise between spectral resolution and anticipated computational burden, which scales with the number of channels. While in principle the line emission could be analyzed at higher spectral resolution with less aggressive binning factors, the considerable line width of UZ Tau E means that the disk rotation curve is still sampled with more than 30 effective channels at the chosen resolution.

We produced a final image of the dust continuum of UZ Tau E and W using the multiscale, multifrequency clean algorithm and Briggs parameter robust $=0.5$ (see Figure 2). We fit the dust emission of UZ Tau E with an elliptical Gaussian using the uvmodelfit task and find a flux density of $145.72 \pm 0.04 \mathrm{mJy}$ (the uncertainty does not include systematic calibration uncertainties). The dust emission from 


\section{APPENDIX}

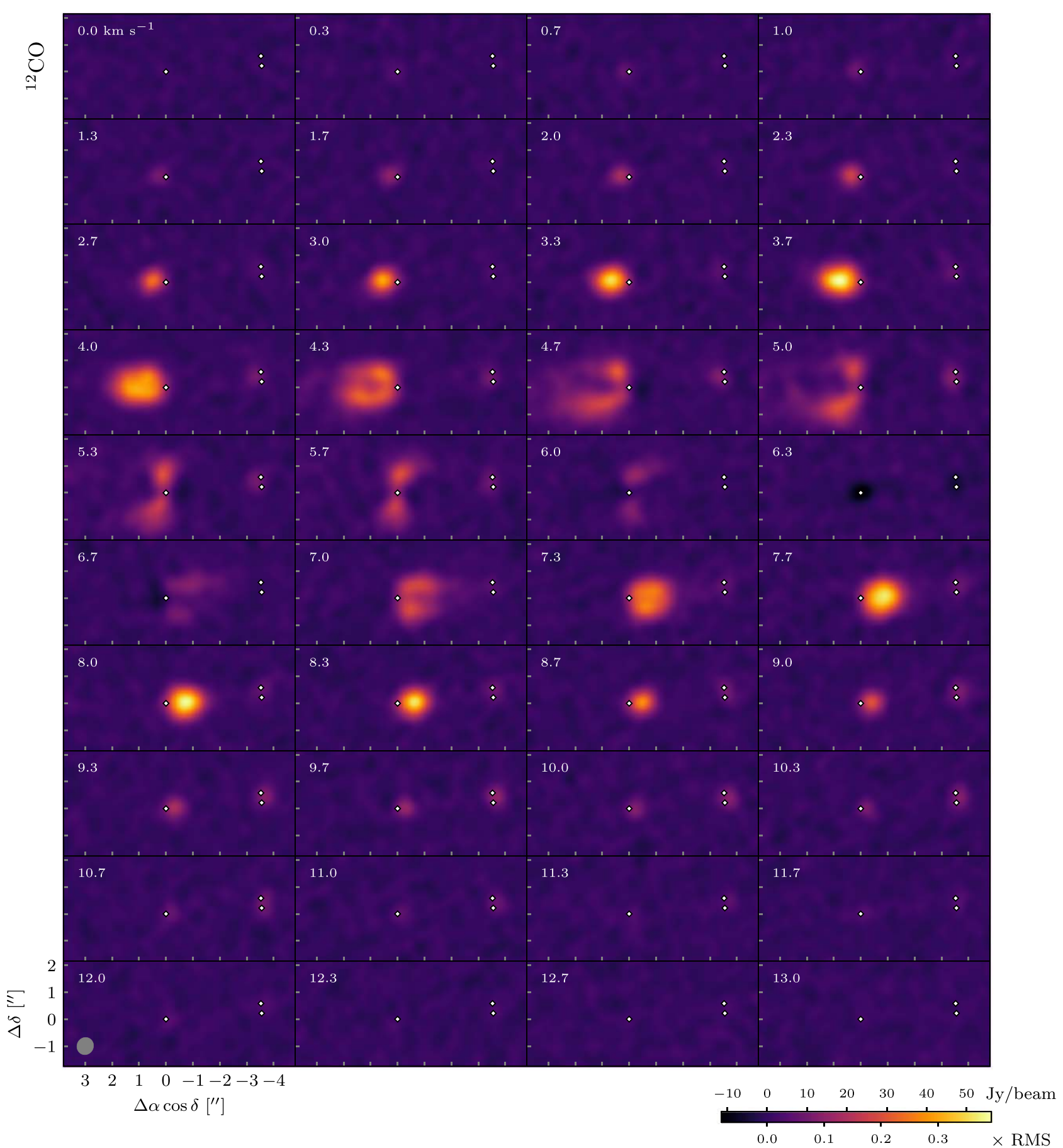

Figure 3. Channel maps for ${ }^{12} \mathrm{CO}$. The positions of UZ Tau E, Wa, and $\mathrm{Wb}$ are marked as in Figure 2. All velocities are in the LSRK frame.

the western component is extended along a north-south axis. Recently, Tripathi et al. (2018) published Karl G. Jansky Very Large Array (JVLA) observations at 30.5 and $37.5 \mathrm{GHz}$ with 0 "! 1 resolution and individually resolved the $\mathrm{Wa}$ and $\mathrm{Wb}$ circumstellar disks, showing that at these frequencies they are near equal brightness. Although we are unable to individually resolve the $\mathrm{Wa}$ and $\mathrm{Wb}$ components with the 0 ". 6 beam of our observations, the elongated emission is indicative that we are seeing emission coming from both the $\mathrm{Wa}$ and $\mathrm{Wb}$ components. The total measured flux from these is $34 \mathrm{mJy}$, consistent with the $225 \mathrm{GHz}$
Combined Array for Research in Millimeter-wave Astronomy measurements also presented in Tripathi et al. (2018).

We synthesized images of the CO isotopologue line emission using the CASA tclean task with Briggs robust $=0.5$ weighting. We used the auto-multithresh to generate an initial starting mask for each CO isotopologue, which was then edited by hand channel by channel to conform to the observed emission from the $\mathrm{E}$ and $\mathrm{W}$ components. The properties of the synthesized images are summarized in Table 1, and the channel maps are shown in Figures 3-5. 

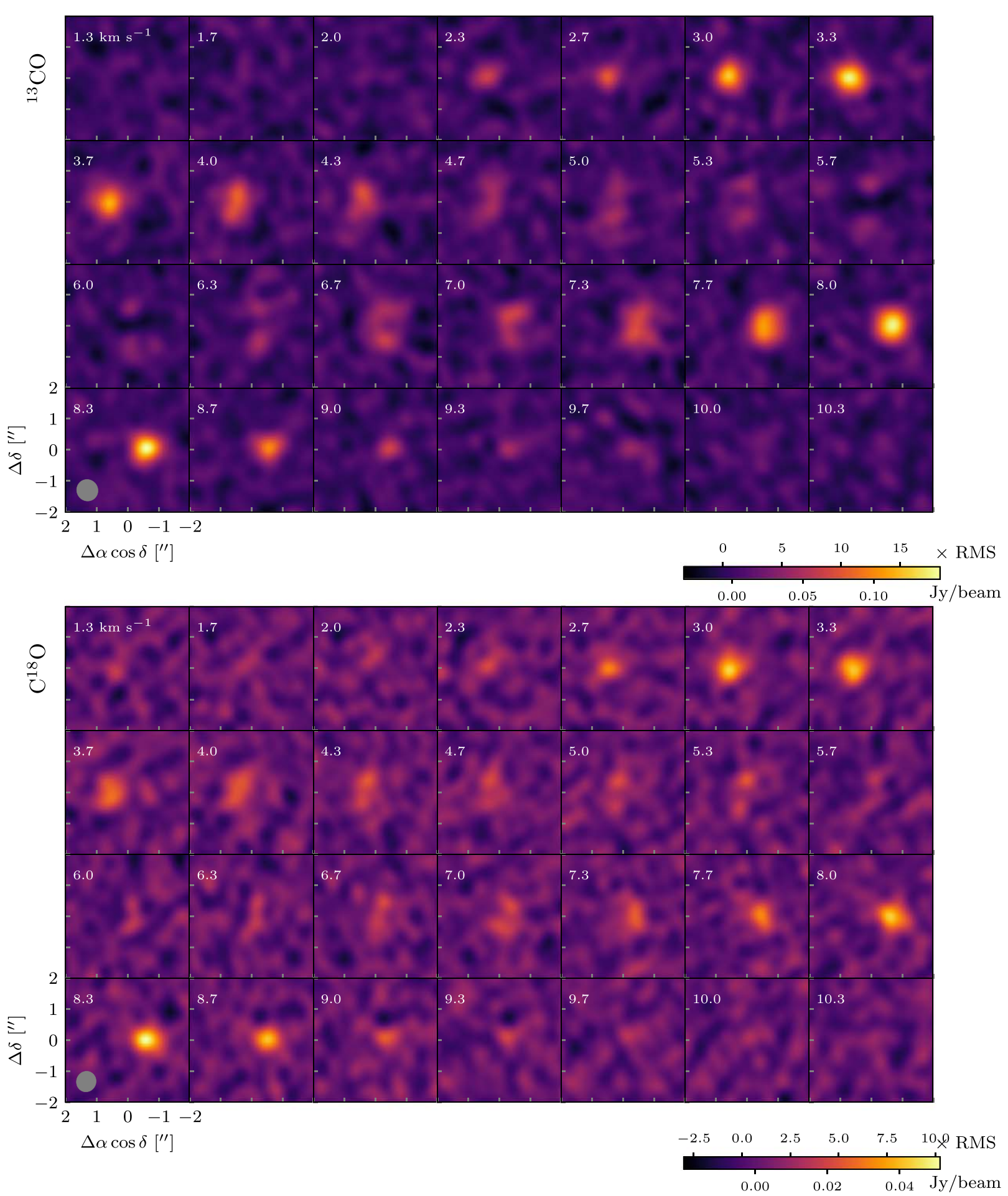

Figure 4. Channel maps for ${ }^{13} \mathrm{CO}$ and $\mathrm{C}^{18} \mathrm{O}$ isotopologues. The maps are shown centered on UZ Tau E because the emission from UZ Tau W is not evident in individual frames. All velocities are in the LSRK frame.

Spatially resolved channel maps can be useful for determining the systemic velocity of a protoplanetary disk because they generally exhibit reflective symmetry across the disk minor axis, about the systemic velocity. The central channel at the systemic velocity contains two lobes in a characteristic "figure eight" shape, while lobes in redshifted or blueshifted channels stretch into "C-like" shapes. Based on visual inspection of the ${ }^{12} \mathrm{CO}$ maps synthesized at the highest $79 \mathrm{~m} \mathrm{~s}^{-1}$ native resolution (see the Figure 5), it is clear that the UZ Tau E "figure
Table 1

ALMA Image Properties

\begin{tabular}{|c|c|c|}
\hline & Beam Dimensions, P.A. & rms $\left(\mathrm{mJy}\right.$ beam $\left.^{-1}\right)$ \\
\hline $233 \mathrm{GHz}$ cont. & $0 " .66 \times 0 . " 61,-25^{\circ}$ & 0.070 \\
\hline${ }^{12} \mathrm{CO} J=2-1$ & $0 ! \prime 65 \times 0 ! " 61,-26^{\circ}$ & 7 \\
\hline${ }^{13} \mathrm{CO} J=2-1$ & $0 " .70 \times 0.68,35^{\circ}$ & 8 \\
\hline $\mathrm{C}^{18} \mathrm{O} J=2-1$ & $0 " .66 \times 0 . " 63,-19^{\circ}$ & 5 \\
\hline
\end{tabular}

Note. The rms noise levels recorded for the spectral line cubes correspond to the values per $334 \mathrm{~m} \mathrm{~s}^{-1}$ channel. All images were synthesized with robust $=0.5$. 


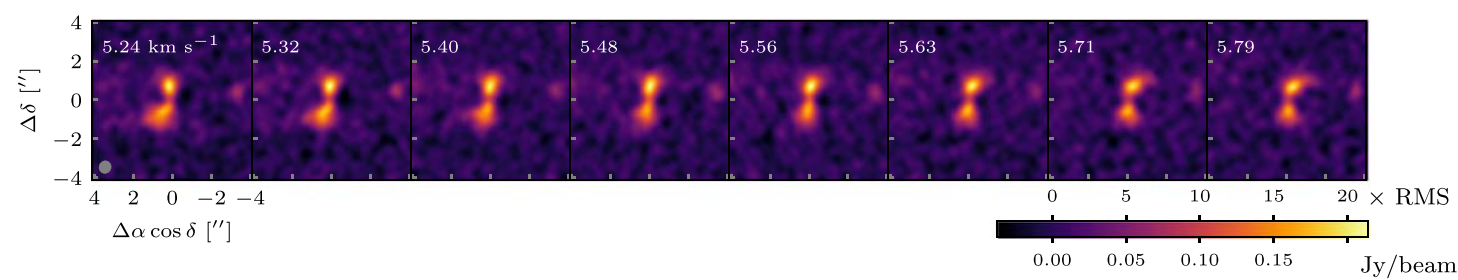

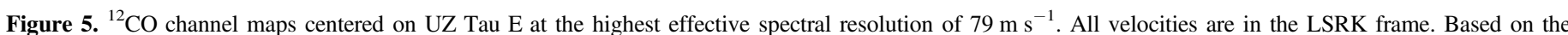

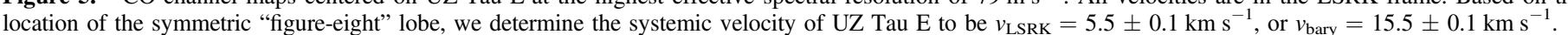

eight" is located at $v_{\mathrm{LSRK}}=5.5 \pm 0.1 \mathrm{~km} \mathrm{~s}^{-1}\left(v_{\text {bary }}=15.5 \pm\right.$ $\left.0.1 \mathrm{~km} \mathrm{~s}^{-1}\right) .{ }^{10}$ The ${ }^{12} \mathrm{CO}$ channel maps in Simon et al. (2000) appear consistent with our determination of the systemic velocity-their coarsely spaced channel maps $\left(1.2 \mathrm{~km} \mathrm{~s}^{-1}\right)$ show the "figure eight" at or near $v_{\text {LSRK }}=5.4 \mathrm{~km} \mathrm{~s}^{-1}$. By forward-modeling single-dish $\mathrm{CN}$ observations, Guilloteau et al. (2013) obtained a systemic velocity of $v_{\mathrm{LSRK}}=5.96 \pm$ $0.47 \mathrm{~km} \mathrm{~s}^{-1}$, which is consistent with our determination given the large uncertainty on their measurement. Guilloteau et al. (2013) also determined a systemic velocity by analyzing the wings of ${ }^{13} \mathrm{CO}$ emission, which they argued should be minimally affected by cloud contamination, and obtained $v_{\text {LSRK }}=5.90 \pm 0.18 \mathrm{~km} \mathrm{~s}^{-1}$. It is unclear how cloud-contaminated channels were identified and masked in this analysis, and so it is possible that this systemic velocity measurement may have been affected by residual cloud contamination. Our systemic velocity is mildly discrepant $(2 \sigma)$ from that determined spectroscopically $\left(\gamma_{\text {LSRK }}=3.9 \pm 0.7 \mathrm{~km} \mathrm{~s}^{-1}\right.$; Jensen et al. 2007). This difference likely originates from uncalibrated RV zero-point offsets, which are frequently of this magnitude and spectrograph dependent.

Deviations from the reflective symmetry of $\mathrm{CO}$ channel maps - most visible as "missing" flux_-indicate that the UZ Tau line emission is contaminated by a foreground molecular cloud. We generated moment- 0 maps (total intensity integrated over all velocity channels) for the ${ }^{12} \mathrm{CO},{ }^{13} \mathrm{CO}$, and $\mathrm{C}^{18} \mathrm{O}$ isotopologues using the immoments CASA task summing across all spectral channels in the range $0.0-13.0 \mathrm{~km} \mathrm{~s}^{-1}$ LSRK. We detect faint line emission from UZ Tau W in all CO isotopologues (see Figure 2). Using the moment- 0 maps for each isotopologue as a guide, we created new masks encapsulating the full spatial extent of the emission, for each transition, for both UZ Tau E and W. In contrast to the CLEAN masks used to synthesize the channel maps, which have different shapes for each channel, these masks were the same shape for all channels. We calculated the total integrated flux for the lines using these masks and the imstat task on the moment- 0 maps. For UZ Tau E, the ${ }^{12} \mathrm{CO},{ }^{13} \mathrm{CO}$, and $\mathrm{C}^{18} \mathrm{O}$ line fluxes are $6.01 \pm 0.02 \mathrm{Jy} \mathrm{km} \mathrm{s}^{-1}, \quad 0.99 \pm$ $0.02 \mathrm{Jy} \mathrm{km} \mathrm{s}^{-1}$, and $0.35 \pm 0.01 \mathrm{Jy} \mathrm{km} \mathrm{s}^{-1}$, respectively. For $\mathrm{UZ} \mathrm{Tau} \mathrm{W}$, the line fluxes are $0.77 \pm 0.02 \mathrm{Jy} \mathrm{km} \mathrm{s}^{-1}, 0.13 \pm$ $0.02 \mathrm{Jy} \mathrm{km} \mathrm{s}^{-1}$, and $0.06 \pm 0.01 \mathrm{Jy} \mathrm{km} \mathrm{s}^{-1}$, respectively. We note that because of the foreground cloud contamination, these line fluxes are lower limits only.

To confidently identify which specific channels suffer cloud contamination and excise them from the dynamical analysis, we inspected the ${ }^{12} \mathrm{CO}$ and ${ }^{13} \mathrm{CO} J=1-0$ maps of the Five College Radio Astronomy Observatory CO Mapping Survey of the Taurus Molecular Cloud (Goldsmith et al. 2008; Narayanan et al. 2008). This survey was carried out with the $13.7 \mathrm{~m}$ single-dish

\footnotetext{
10 In the direction of UZ Tau, the conversion between the kinematic local standard of rest (LSRK) and the barycentric velocity is $v_{\text {bary }}=v_{\mathrm{LSRK}}+10.00 \mathrm{~km} \mathrm{~s}^{-1}$.
}

Quabbin telescope, and the maps have a spatial resolution of $45^{\prime \prime}$ and velocity resolution of $1 \mathrm{~km} \mathrm{~s}^{-1}$. At the location of the UZ Tau system, we find that there is significant ${ }^{12} \mathrm{CO}$ and ${ }^{13} \mathrm{CO}$ cloud emission for channels in the velocity range $v_{\text {LSRK }}=4-7 \mathrm{~km} \mathrm{~s}^{-1}$ and a faint trace of emission in the $7-8 \mathrm{~km} \mathrm{~s}^{-1}$ channel. Therefore, we choose to mask from our analysis all channels in the range $4.0 \mathrm{~km} \mathrm{~s}^{-1} \leqslant v_{\mathrm{LSRK}} \leqslant$ $7.5 \mathrm{~km} \mathrm{~s}^{-1}$.

Using the quadratic fit technique implemented by Teague \& Foreman-Mackey (2018), we made maps of the velocity field of the UZ Tau system as probed by ${ }^{12} \mathrm{CO}$ emission (see Figure 6). The velocity field of the combined emission of UZ Tau W has a nodal position angle $\Omega_{\text {disk }}$ that approximately matches that of UZ Tau E. Given the Tripathi et al. (2018) observations, we know that $\mathrm{Wa}$ and $\mathrm{Wb}$ host individual circumstellar dust disks. The emission we see is consistent with both of these stars hosting gas disks. Higher spatial resolution observations in ${ }^{12} \mathrm{CO}$ or other dense-gas tracers would definitively associate the western gas emission with $\mathrm{Wa}$ and/or $\mathrm{Wb}$, measure the inclination of the $\mathrm{Wa}$ and $\mathrm{Wb}$ disks, and permit a measurement of coplanarity between the $\mathrm{E}$ and $\mathrm{Wa}$ and $\mathrm{Wb}$ disks.

Notwithstanding the potential biases from cloud contamination, it is still worthwhile to compare the velocity extent of the UZ Tau E and W emission. From Figure 6, it is clear that one or both of the $\mathrm{W}$ disks host emission at larger RVs than the $\mathrm{E}$ disk. The line width of the emission is relatively large considering that the $\mathrm{Wa}$ and $\mathrm{Wb}$ stars are less than half of the mass of the E spectroscopic binary. This would seem to indicate that either the $\mathrm{Wa}$ and $\mathrm{Wb}$ disks are observed at higher inclination, their stellar host masses are greater than their spectral type would seem to imply, or both.

Hartmann et al. (1986) used optical spectroscopic observations of $\mathrm{W}$ to determine a systemic velocity of $v_{\mathrm{LSRK}}=8.5 \mathrm{~km} \mathrm{~s}^{-1}$ $\left(v_{\text {bary }}=18.5 \mathrm{~km} \mathrm{~s}^{-1}\right)$; however, in that analysis, the binary nature of $\mathrm{W}$ was not yet known. Taken at face value, given the large separation on the sky between the $\mathrm{E}$ and $\mathrm{W}$ systems, the $\Delta v_{r} \approx 3 \mathrm{~km} \mathrm{~s}^{-1} \mathrm{RV}$ discrepancy between $\mathrm{E}$ and $\mathrm{W}$ would be suggestive that the two binary systems are not gravitationally bound to each other. However, given the proximity of $\mathrm{E}$ and $\mathrm{W}$ and the considerable uncertainty in the RV of W (E. L. N. Jensen 2019, private communication), the possibility remains that the systems are bound.

\subsection{UZ Tau $E^{13} \mathrm{CO}$ and $\mathrm{C}^{18} \mathrm{O}$ Dynamical Analysis}

To determine the total mass $M_{\mathrm{tot}}=M_{\mathrm{Ea}}+M_{\mathrm{Eb}}$ of the UZ Tau E spectroscopic binary, we model the rotation of the circumbinary disk as traced by molecular line emission. Previously, we noted that the UZ Tau system suffers from cloud contamination. Because ${ }^{12} \mathrm{CO}$ is the isotopologue most readily affected by cloud contamination, we take a conservative approach and only model the emission from the less abundant 


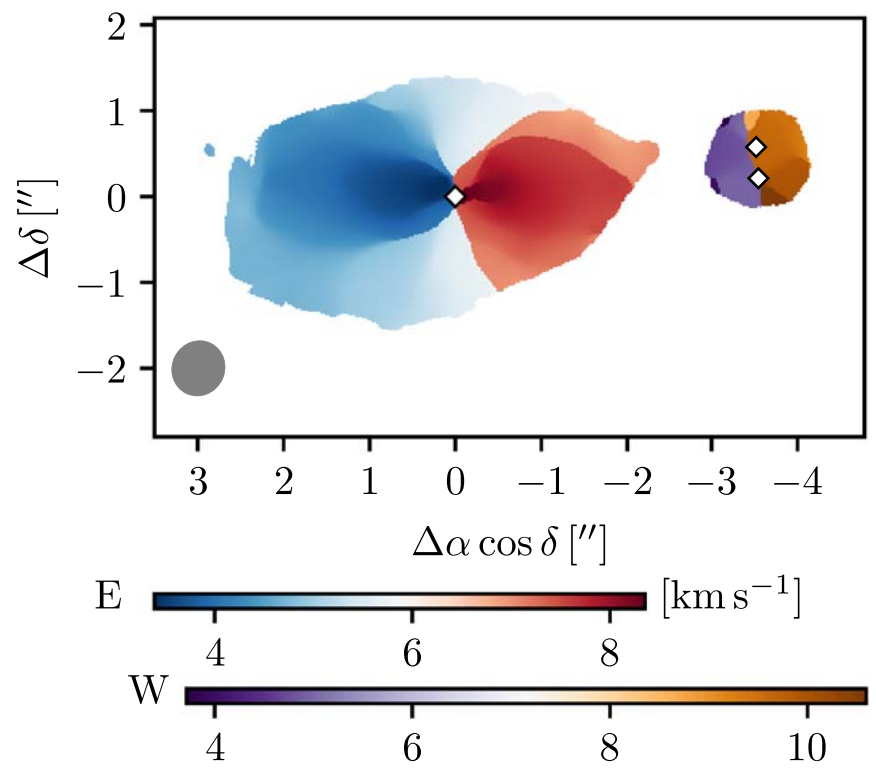

Figure 6. Velocity field of the UZ Tau system, generated from the ${ }^{12} \mathrm{CO}$ emission using a quadratic fit as implemented in Teague \& ForemanMackey (2018) and masking all zeroth-moment pixels below the fifth intensity percentile. The color bars indicate the inferred velocity at each pixel for both UZ Tau E and UZ Tau W and are stretched independently. We caution that, due to cloud contamination, the retrieved velocities may be partially biased in the spatial pixels containing emission at $v_{\mathrm{LSRK}}=4.0-7.5 \mathrm{~km} \mathrm{~s}^{-1}$ (see Figure 3). The western emission is consistent with originating from two small circumstellar disks around $\mathrm{UZ}$ Tau $\mathrm{Wa}$ and $\mathrm{Wb}$, as seen in $\sim 30 \mathrm{GHz}$ dust emission by Tripathi et al. (2018).

${ }^{13} \mathrm{CO}$ and $\mathrm{C}^{18} \mathrm{O}$ isotopologues in channels outside of the contaminated velocity ranges $\left(v_{\mathrm{LSRK}}=4.0-7.5 \mathrm{~km} \mathrm{~s}^{-1}\right)$.

Briefly, our dynamical mass technique works by forwardmodeling the molecular emission directly to the visibility plane. The disk structure is set by a temperature power law (with exponent $q$ and normalization at 10 au by $T_{10}$ ) and a surface density power law (exponent $\gamma=1$ ) with an exponential taper outside of the disk characteristic radius, $r_{c}$. The density power law is normalized by the total disk mass $\left(M_{\text {disk }}\right)$. For a given radius, the disk is assumed to be vertically isothermal and the vertical density distribution set by hydrostatic equilibrium. The velocity field of the disk is assumed to be Keplerian and entirely determined by the central stellar mass $\left(M_{\text {tot }}\right)$. We assume the distance to UZ Tau $\mathrm{E}$ to be $d=131.2 \mathrm{pc}$. The disk structure is oriented relative to the observer based on geometric parameters (inclination relative to the sky tangent plane $i_{\text {disk }}$, position angle of the ascending node $\Omega_{\text {disk }}$, and offsets from the phase center $\delta_{\alpha}$ and $\delta_{\delta}$ ), and then channel maps are ray-traced using the RADMC-3D software package (Dullemond 2012). The channel maps are then Fourier transformed, sampled at the baselines corresponding to the array position during the observations, and compared to the visibilities with a complex-valued $\chi^{2}$ likelihood function. After incorporating uniform priors on the disk structure parameters and a geometrical prior on the disk inclination, the posterior distribution is sampled using our Julia implementation of the Markov chain Monte Carlo (MCMC) affine-invariant ensemble sampler (Goodman \& Weare 2010; Foreman-Mackey et al. 2013). For more information on the implementation of these procedures see Czekala et al. (2015) and the DiskJockey codebase. ${ }^{11}$

\footnotetext{
11 https://github.com/iancze/DiskJockey
}

Table 2

Inferred Disk Model Parameters

\begin{tabular}{lcc}
\hline \hline Parameter & ${ }^{13} \mathrm{CO}$ & $\mathrm{C}^{18} \mathrm{O}$ \\
\hline$M_{\text {tot }}\left(M_{\odot}\right)$ & $1.19 \pm 0.01$ & $1.23 \pm 0.01$ \\
$r_{c}(\mathrm{au})$ & $28 \pm 2$ & $24 \pm 2$ \\
$T_{10}(\mathrm{~K})$ & $48 \pm 1$ & $28 \pm 1$ \\
$q$ & $0.5^{\mathrm{a}}$ & $0.5^{\mathrm{a}}$ \\
$\log _{10} M_{\text {disk }} \log _{10}\left(M_{\odot}\right)$ & $-5.77 \pm 0.08$ & $-5.3 \pm 0.1$ \\
$\xi\left(\mathrm{km} \mathrm{s}^{-1}\right)$ & $0.23 \pm 0.02$ & $0.19 \pm 0.02$ \\
$i_{\text {disk }}(\mathrm{deg})$ & $56.15^{\mathrm{a}}$ & $56.15^{\mathrm{a}}$ \\
$\Omega_{\text {disk }}(\mathrm{deg})$ & $269.9 \pm 0.4$ & $269.3 \pm 0.4$ \\
$v_{r}\left(\mathrm{~km} \mathrm{~s}^{-1}\right)$ & $5.74 \pm 0.01^{\mathrm{b}}$ & $5.71 \pm 0.01^{\mathrm{b}}$ \\
$\delta_{\alpha}(\operatorname{arcsec})$ & $0.79 \pm 0.004$ & $0.78 \pm 0.004$ \\
$\delta_{\delta}(\operatorname{arcsec})$ & $-0.21 \pm 0.004$ & $-0.21 \pm 0.004$ \\
\hline
\end{tabular}

Notes. The 1D marginal posteriors are well described by a Gaussian, so we report symmetric error bars here (statistical uncertainties only). These parameters were inferred using a distance of $d=131.2 \mathrm{pc}$.

a Parameter is fixed.

${ }^{\mathrm{b}}$ LSRK reference frame.

Most molecular line observations of disks leave a degeneracy as to whether $i_{\text {disk }}<90^{\circ}$ or $i_{\text {disk }}>90^{\circ}$ (orbits moving counterclockwise or clockwise on the sky plane, respectively). Although gas observations reveal instantaneous line-of-sight velocity information throughout the entire disk, they do not provide the sense of disk rotation in the same manner as astrometric binary orbits. The inclination degeneracy can sometimes be broken with very sensitive molecular observations, since the brighter of the two lobes of the figure eight at the systemic velocity indicates the far side of the disk (Rosenfeld et al. 2013). For UZ Tau E, the brightness asymmetry seen in the high-resolution ${ }^{12} \mathrm{CO}$ channel maps (see Figure 5) indicates that $i_{\text {disk }}<90^{\circ}$. If there are spatially resolved scattered light images of the disk, the strong forward scattering of micron-sized dust grains can also indicate the near side of the disk. Since the high-resolution observations by Long et al. (2018) of the dust continuum yield a more precise constraint on the disk inclination $\left(i_{\text {disk }}=56^{\circ} .15 \pm 1.5\right)$ than can be expected from our lower-resolution data, we fix the disk inclination to this value in order to reduce the parameter space of our model and speed convergence of the MCMC ensemble.

We experimented with various temperature profiles. Our first experiments letting the normalization and exponent float yielded flat $(q \approx 0)$ and cold $\left(T_{10} \approx 12 \mathrm{~K}\right)$ temperature profiles for both ${ }^{13} \mathrm{CO}$ and $\mathrm{C}^{18} \mathrm{O}$. We then tried two temperature profiles with fixed power-law exponents of $q=0.50$ and $q=0.75$, which are common values for flared and geometrically thin disks, respectively. The choice of $q=0.50$ yielded the most reasonable temperature normalization $\left(T_{10} \approx 50 \mathrm{~K}\right)$ and a temperature profile consistent with the dust temperature profile presented in Long et al. (2018). The full constraints on the model parameters using the ${ }^{13} \mathrm{CO}$ and $\mathrm{C}^{18} \mathrm{O}$ transitions are listed in Table 2. From the ${ }^{13} \mathrm{CO}$ and $\mathrm{C}^{18} \mathrm{O}$ isotopologues, the total stellar mass is inferred to be $M_{\text {tot }}=1.19 \pm 0.01 M_{\odot}$ and $M_{\text {tot }}=1.23 \pm 0.01 M_{\odot}$, respectively (statistical errors only). Because these measurements are made with independent data sets but are statistically inconsistent with each other, this indicates that there is at least a $0.02 M_{\odot}$ systematic error affecting these results, which is probably a result of missing complexity in our disk structure model (e.g., a vertical temperature gradient, $\mathrm{CO}$ freezeout). The data, model, and residual channel maps for the ${ }^{12} \mathrm{CO}$ and ${ }^{13} \mathrm{CO}$ transitions are 
shown in Appendix Figures 15 and 16, respectively. We average these two results and add the uncertainties in quadrature to obtain a combined mass constraint of $M_{\text {tot }}=1.21 \pm$ $0.02 M_{\odot}$.

Because we fixed $i_{\text {disk }}$ and the distance to UZ Tau E in these analyses, there are two more sources of statistical uncertainty on $M_{\text {tot }}$ to be accounted for. As the spatial resolution of the observations degrades, the measurements of $M_{\mathrm{tot}}$ and $i_{\text {disk }}$ begin to correlate along the $M_{\text {tot }} \sin ^{2} i_{\text {disk }}$ disk rotation curve degeneracy. To reintroduce the uncertainty associated with $i_{\text {disk }}$ onto the inference of $M_{\text {tot }}$, we translate the $\sigma_{\text {idisk }}=1.5$ Gaussian inclination uncertainty from Long et al. (2018) into an $M_{\text {tot }}$ uncertainty along the $M_{\mathrm{tot}} \sin ^{2} i_{\text {disk }}$ degeneracy, yielding $\sigma_{M \text { tot }, i}=0.05 M_{\odot}$. The uncertainty in the distance to the system $(d=131.2 \pm 1.7 \mathrm{pc})$ translates linearly into an uncertainty in the total mass $\left(\sigma_{M \text { tot }, d}=0.01 M_{\odot}\right)$. These two uncertainties are added in quadrature to deliver a final mass determination of $M_{\text {tot }}=1.21 \pm 0.05 M_{\odot}$. This value agrees well with the Simon et al. (2000) analysis using ${ }^{12} \mathrm{CO}$, which obtained $M_{\mathrm{tot}}=$ $1.22 \pm 0.07 M_{\odot}$ (scaled to $d=131.2 \mathrm{pc}$ ). Throughout all of the choices of temperature power law (floating, $q=0.50$, and $q=0.75)$, the dynamical mass remained the same for each transition within statistical uncertainties.

UZ Tau E was first discovered as a single-lined spectroscopic binary by Mathieu et al. (1996). Using high-resolution infrared spectroscopy, Prato et al. (2002) revealed UZ Tau E as a double-lined spectroscopic binary (SB2). Jensen et al. (2007) further refined the orbital parameters using additional RV observations, including those acquired by Martín et al. (2005). Without needing to know the distance to the system, doublelined RV solutions yield the mass ratio of the stars $(q=$ $\left.M_{B} / M_{A}\right)$ and the quantity $M_{\mathrm{tot}} \sin ^{3} i_{\star}$. Since the protoplanetarydisk-based technique independently measures $M_{\text {tot }}$, we can combine these two results to solve directly for $i_{\star}$. For UZ Tau E, Jensen et al. (2007) find $M_{\text {tot }} \sin ^{3} i_{\star}=0.69 \pm$ $0.13 M_{\odot}$, and so we infer the binary inclination relative to the sky plane to be $i_{\star}=56^{\circ} .1 \pm 5^{\circ} .7$ (technically, there is also a degenerate solution with $\left.i_{\star}=123^{\circ} .9 \pm 5^{\circ} .7\right)$. Using the Jensen et al. (2007) mass ratio of $q=0.30 \pm 0.03$, the individual stellar masses are $M_{\mathrm{Ea}}=0.93 \pm 0.04 M_{\odot}$ and $M_{\mathrm{Eb}}=0.28 \pm$ $0.02 M_{\odot}$.

As we noted in the Introduction, calculating the mutual inclination between the binary orbit and the circumbinary disk requires knowledge of $\Omega_{\star}$. Including UZ Tau E, there are now four systems with $i_{\text {disk }} \simeq i_{\star}$ but for which our ignorance of $\Omega_{\star}$ prevents a direct calculation of mutual inclinations. Nevertheless, we can make statistical statements, which, as we show in the next section, are constraining.

\section{The Mutual Inclinations of Circumbinary Disks}

In this section, we first compile all circumbinary protoplanetary and debris disks in the literature. Then, we estimate the mutual inclination $\theta$ of these systems. For some systems, $\theta$ can be calculated directly via Equation (1), whereas other systems require an indirect approach. For the CB disks around SB2s in particular, we show that a naïve estimate of mutual inclination is biased, so we implement a hierarchical Bayesian model to infer the mutual inclination distribution of this subsample. Finally, we examine how the mutual inclination distribution changes with binary orbital period across the full sample.

\subsection{The Circumbinary Disk Sample}

The majority of protoplanetary CB disks were identified in two ways. First, there are those that originated from RV surveys for spectroscopic binaries in star-forming regions (e.g., Mathieu 1994; Melo 2003; Guenther et al. 2007). These RV searches were primarily sensitive to binaries with orbits shorter than $1 \mathrm{yr}$ (semimajor axes smaller than $1 \mathrm{au}$ ). Some fraction of the binary stars discovered by these surveys were found to have spectroscopic accretion signatures and infrared and millimeter excesses above the stellar photosphere. The disks around these binaries were targeted with submillimeter interferometers (e.g., Simon et al. 2000; Rosenfeld et al. 2012; Czekala et al. 2015) or high-contrast imagers (Ginski et al. 2018).

Second, there are the protoplanetary CB disks that originated from high-contrast adaptive optics and/or nonredundant masking searches for binary stars and planetary-mass companions in star-forming regions (e.g., Ireland \& Kraus 2008; Ruíz-Rodríguez et al. 2016). Current instrumentation has enabled observations that probe binary orbits with separations as small as $10 \mathrm{au}$. These techniques, in addition to infrared interferometry (Schaefer et al. 2018), are also used to monitor the orbital motions of binary stars and companions. In many cases, spatially resolved submillimeter observations of the discovered binary sources were independently acquired by surveys of these same star-forming regions (Cox et al. 2017).

A third, smaller sample of protoplanetary CB disks are those identified indirectly through light-curve analysis. Long-term photometric monitoring campaigns of $\mathrm{T}$ Tauri stars discovered some sources with periodic, evolving dips (e.g., KH 15D; Herbst et al. 2002; Winn et al. 2003; Johnson \& Winn 2004). Modeling of these systems indicated that an optically thick, inclined circumbinary disk was responsible for screening the stars (Chiang \& Murray-Clay 2004; Plavchan et al. 2008). There is also one EB whose variable light curve shows evidence for a circumbinary disk (Gillen et al. 2014, 2017). The remainder of the protoplanetary $\mathrm{CB}$ disks were identified by miscellaneous means, often serendipitously from detailed studies of individual targets.

The sample of circumbinary debris disks was acquired in much the same way as the protoplanetary disk sample, with disk discoveries primarily coming from surveys by the Herschel satellite at far-IR and submillimeter wavelengths (Matthews et al. 2010) and binarity follow-up observations with adaptive optics. There are a few nearby resolved CB debris disks (e.g., Kennedy et al. 2012a, 2012b; Kennedy 2015); however, the modest spatial resolution of Herschel means that most debris disks known to be circumbinary are spatially unresolved (Rodriguez et al. 2015).

We separate the full collection of CB disk systems into three categories based on the fidelity of their measured parameters and their ability to inform our study of the distribution of mutual inclinations. The first group contains the circumbinary systems that have precise measurements of the disk and stellar orientations (Table 3 ) and includes six protoplanetary disks and four debris disks. The second group contains protoplanetary systems that may have partial stellar orbits and/or ambiguities in their disk orientations, which leads to moderate uncertainties in mutual inclinations (Table 4). The third group contains those protoplanetary systems that are known to be circumbinary but do not have complete orbit information and/or a spatially resolved disk observation, and so no mutual inclination can be calculated (Table 5). We include these systems here in the hope 
Table 3

CB Disks around Binary Stars with Precisely Measured Orbital Parameters

\begin{tabular}{|c|c|c|c|c|c|c|c|c|c|c|c|c|c|}
\hline Name & $\begin{array}{c}P \\
\text { (days) }\end{array}$ & $\begin{array}{c}M_{1} \\
\left(M_{\odot}\right)\end{array}$ & $\begin{array}{c}M_{2} \\
\left(M_{\odot}\right)\end{array}$ & $q$ & $\begin{array}{c}a \\
(\mathrm{au})\end{array}$ & $e$ & $\begin{array}{c}i_{\text {disk }} \\
(\mathrm{deg})\end{array}$ & $\begin{array}{l}\Omega_{\text {disk }} \\
(\mathrm{deg})\end{array}$ & $\begin{array}{c}i_{\star} \\
(\mathrm{deg})\end{array}$ & $\begin{array}{c}\Omega_{\star} \\
(\operatorname{deg})\end{array}$ & $\begin{array}{c}\theta \\
(\mathrm{deg})\end{array}$ & $\begin{array}{l}\text { Age } \\
\text { (Myr) }\end{array}$ & References \\
\hline V4046 Sgr ${ }^{\mathrm{a}}$ & 2.4 & 0.9 & 0.85 & 0.94 & 0.04 & 0.00 & $33.5 \pm 1.4$ & $256 \pm 1$ & $33.4 \pm 0.6$ & $\cdots$ & $<2.3^{\mathrm{b}}$ & 13 & (1), (2), (3), (4) \\
\hline CoRoT 2239 & 3.9 & 0.67 & 0.495 & 0.74 & 0.05 & 0.00 & $81 \pm 5$ & $\ldots$ & $85.09 \pm 0.15$ & $\cdots$ & $<5$ & 3 & $(5),(6),(7)$ \\
\hline AK Sco & 13.6 & 1.25 & 1.25 & 1.00 & 0.16 & 0.47 & $109.4 \pm 0.5$ & $51.1 \pm 0.3$ & $108.8 \pm 2.4$ & $48 \pm 3$ & $<2.7^{\mathrm{b}}$ & 18 & (8), (9), (10) \\
\hline DQ Tau & 15.8 & 0.63 & 0.59 & 0.94 & 0.15 & 0.57 & $160 \pm 3$ & $4.2 \pm 0.5$ & $158.2 \pm 2.8$ & $\ldots$ & $<2.7^{\mathrm{b}}$ & 3 & (11) \\
\hline $\mathrm{UZ}$ Tau $\mathrm{E}^{\mathrm{a}}$ & 19.1 & 1.02 & 0.29 & 0.29 & 0.15 & 0.33 & $56.15 \pm 1.5$ & $269.6 \pm 0.5$ & $56.1 \pm 5.7$ & $\cdots$ & $<2.7^{\mathrm{b}}$ & 3 & $(12),(13),(14)$ \\
\hline HD $98800 B^{a}$ & 315.0 & 0.7 & 0.6 & 0.86 & 1.05 & 0.78 & $154 \pm 1$ & $197 \pm 2$ & $67 \pm 3$ & $157.6 \pm 2.4$ & $92 \pm 3$ & 10 & $(15),(16),(17),(18)$ \\
\hline HD 131511 & 11.5 & 0.79 & 0.45 & 0.57 & 0.19 & 0.51 & $90 \pm 10$ & $245 \pm 5$ & $93.4 \pm 4.2$ & $248 \pm 3.6$ & $<15$ & $10^{3}$ & (19) \\
\hline$\alpha \mathrm{CrB}$ & 17.4 & 2.58 & 0.92 & 0.36 & 0.20 & 0.37 & $90 \pm 10$ & $345 \pm 20$ & $88.2 \pm 0.1$ & $330 \pm 20$ & $<35$ & 730 & (20) \\
\hline$\beta$ Tri & 31.4 & 3.5 & 1.4 & 0.40 & 0.31 & 0.43 & $130 \pm 10$ & $247 \pm 10$ & $130.0 \pm 0.5$ & $245.2 \pm 0.67$ & $<14$ & 350 & (20) \\
\hline $99 \mathrm{Her}$ & $56.3 \mathrm{yr}$ & 0.94 & 0.46 & 0.49 & 16.58 & 0.77 & $45 \pm 5$ & $72 \pm 10$ & $39 \pm 2$ & $41 \pm 2$ & $80 \pm 6$ & $6 \times 10^{3}$ & (21) \\
\hline
\end{tabular}

Notes. Mutual inclination $\theta$ upper limits enclose $68 \%$ of the posterior probability distribution. Protoplanetary disks are above the horizontal rule and debris disks are below it.

${ }^{a}$ Hosts a binary companion orbiting beyond the circumbinary disk.

${ }^{\mathrm{b}}$ Mutual inclination inferred via hierarchical Bayesian model.

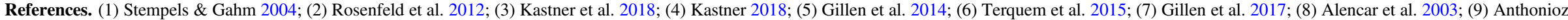

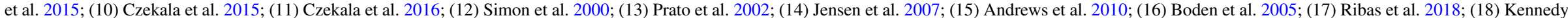
et al. 2019; (19) Kennedy 2015; (20) Kennedy et al. 2012a; (21) Kennedy et al. 2012b. 
Table 4

CB Protoplanetary Disks around Stars with Moderate Orientation Uncertainties

\begin{tabular}{|c|c|c|c|c|c|c|c|c|c|c|c|c|c|}
\hline Name & $P$ & $\begin{array}{c}M_{1} \\
\left(M_{\odot}\right)\end{array}$ & $\begin{array}{c}M_{2} \\
\left(M_{\odot}\right)\end{array}$ & $q$ & $\begin{array}{c}a \\
(\mathrm{au})\end{array}$ & $e$ & $\begin{array}{c}i_{\text {disk }} \\
(\mathrm{deg})\end{array}$ & $\begin{array}{l}\Omega_{\text {disk }} \\
(\mathrm{deg})\end{array}$ & $\begin{array}{c}i_{\star} \\
(\mathrm{deg})\end{array}$ & $\begin{array}{c}\Omega_{\star} \\
(\mathrm{deg})\end{array}$ & $\begin{array}{c}\theta \\
(\mathrm{deg})\end{array}$ & $\begin{array}{c}\text { Age } \\
\text { (Myr) }\end{array}$ & References \\
\hline TWA $3 A^{a}$ & 34.9 days & $\ldots$ & $\ldots$ & 0.84 & 0.127 & 0.628 & $43 \pm 10$ & $110 \pm 15$ & $47 \pm 15$ & $108 \pm 15$ & $<25$ & 10 & $(1),(2),(\star)$ \\
\hline GW Ori $A-B^{b}$ & 241.5 days & 2.8 & 1.68 & 0.60 & 1.25 & 0.13 & $137 \pm 2$ & $1 \pm 1$ & $157 \pm 1$ & $263 \pm 13$ & $50 \pm 5$ & 1 & (3) \\
\hline HD 200775 & $3.92 \mathrm{yr}$ & 5.37 & 4.4 & 0.82 & 5 & 0.3 & $55 \pm 1$ & $180 \pm 8^{c}$ & $66 \pm 7$ & $172 \pm 6$ & $<20$ & 0.1 & (4), (5), (6) \\
\hline GW Ori $A B-C^{b}$ & $11.0 \mathrm{yr}$ & 4.48 & 1.15 & 0.26 & 9.2 & 0.13 & $137 \pm 2$ & $1 \pm 1$ & $150 \pm 7$ & $282 \pm 9$ & $45 \pm 5$ & 1 & (3) \\
\hline R CrA & $30 \mathrm{yr}$ & 2 & 0.5 & 0.25 & 29 & 0.4 & $35 \pm 10^{\mathrm{c}}$ & $180 \pm 10^{\mathrm{c}}$ & $70 \pm 15$ & $\ldots$ & $>10$ & 1 & (7), (8) \\
\hline HD 142527 & $50 \mathrm{yr}$ & 2.1 & 0.11 & 0.05 & $<50$ & 0.5 & $153 \pm 1$ & $160.9 \pm 1$ & $125 \pm 5$ & $130 \pm 10^{\mathrm{d}}$ & $35 \pm 5^{\mathrm{d}}$ & 1 & $(9),(10),(11),(12),(13)$ \\
\hline SR $24 N^{a}$ & $111 \mathrm{yr}$ & $\ldots$ & $\ldots$ & & 25 & 0.64 & $121 \pm 7$ & $297 \pm 5$ & $132 \pm 6$ & $72 \pm 4^{\mathrm{e}}$ & $37 \pm 15^{\mathrm{e}}$ & 1 & $(14),(15),(16)$ \\
\hline GG Tau Ab1-Ab2 ${ }^{b}$ & $14 \mathrm{yr}$ & 0.38 & 0.3 & 0.80 & 4.5 & $\ldots$ & $35 \pm 1$ & $277 \pm 0.2$ & $\ldots$ & $\ldots$ & $\ldots$ & 3 & $(17),(18),(19),(20),(21)$ \\
\hline GG Tau Aa-Ab ${ }^{b}$ & $>400 \mathrm{yr}$ & 0.6 & 0.68 & 1.13 & 35 & 0.5 & $143 \pm 1$ & $277 \pm 0.2$ & $132.5 \pm 2$ & $133 \pm 10^{\mathrm{f}}$ & $25 \pm 5^{\mathrm{f}}$ & 3 & $(17),(18),(19),(20),(21)$ \\
\hline IRS 43 & $450 \mathrm{yr}$ & 1 & 1 & 1.00 & 74 & $\ldots$ & $70 \pm 10$ & $90 \pm 5$ & $<30$ & $\ldots$ & $>40$ & $<0.1$ & (22) \\
\hline
\end{tabular}

Notes. Alternative names: TWA 3A = Hen3-600; HD $200775=$ MWC 361; SR 24N = WSB 41

${ }^{\mathrm{a}}$ Hosts a single companion orbiting beyond the circumbinary disk.

${ }^{\mathrm{b}}$ System hosts a circumternary disk.

${ }^{\mathrm{c}}$ Ambiguity exists in the orbit orientation $\left(i<90^{\circ}\right.$ or $\left.i>90^{\circ}\right)$ or $\left(\Omega<180^{\circ}\right.$ or $\left.\Omega>180^{\circ}\right)$.

d There is a $180^{\circ}$ ambiguity in $\Omega_{\star}$ due to lack of RV information, so there is a possible solution with $\Omega_{\star}=310^{\circ} \pm 10^{\circ}$ and $\theta=80^{\circ} \pm 10^{\circ}$.

e There is a $180^{\circ}$ ambiguity in $\Omega_{\star}$ due to lack of RV information, so there is a possible solution with $\Omega_{\star}=252^{\circ} \pm 4^{\circ}$ and $\theta=96^{\circ} \pm 20^{\circ}$.

${ }^{\mathrm{f}}$ There is a $180^{\circ}$ ambiguity in $\Omega_{\star}$ due to lack of RV information, so there is a possible solution with $\Omega_{\star}=313^{\circ} \pm 10^{\circ}$ and $\theta=80^{\circ} \pm 5^{\circ}$.

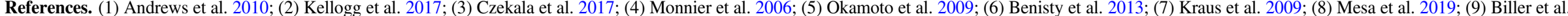

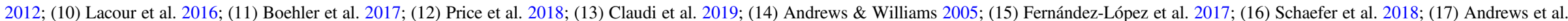
2014; (18) Di Folco et al. 2014; (19) Dutrey et al. 2016; (20) Tang et al. 2016; (21) Cazzoletti et al. 2017; (22) Brinch et al. 2016; ( $\star$ ) I. Czekala et al. 2019, in preparation. 
Table 5

CB Protoplanetary Disks with Unknown Orientations

\begin{tabular}{|c|c|c|c|c|c|c|c|c|c|c|c|c|}
\hline Name & $P$ & $\begin{array}{c}M_{1} \\
\left(M_{\odot}\right)\end{array}$ & $\begin{array}{c}M_{2} \\
\left(M_{\odot}\right)\end{array}$ & $q$ & $\begin{array}{c}a \\
(\mathrm{au})\end{array}$ & $e$ & $\begin{array}{l}i_{\text {disk }} \\
(\mathrm{deg})\end{array}$ & $\begin{array}{l}\Omega_{\text {disk }} \\
(\text { deg) }\end{array}$ & $\begin{array}{c}i_{\star} \\
(\mathrm{deg})\end{array}$ & $\begin{array}{c}\Omega_{\star} \\
(\operatorname{deg})\end{array}$ & $\begin{array}{l}\mathrm{Age} \\
(\mathrm{Myr})\end{array}$ & References \\
\hline HD 104237 & 20 days & 2.2 & 1.4 & 0.64 & 0.22 & 0.6 & $\ldots$ & $\ldots$ & $>90$ & $235 \pm 3$ & 2 & $(1),(2)$ \\
\hline HD $34700 A^{t}$ & 23.5 days & 2 & 2 & 0.99 & 0.69 & 0.25 & 42 & 86 & $\cdots$ & $\cdots$ & 5 & (3), (4) \\
\hline ROXs $42 \mathrm{Ca}^{\mathrm{t}}$ & 36 days & $\ldots$ & $\ldots$ & 0.91 & $\cdots$ & 0.48 & $\ldots$ & $116 \pm 4^{\mathrm{a}}$ & $\ldots$ & $\ldots$ & 2 & $\begin{array}{l}\text { (5), (6), (7), (8), } \\
(9),(10)\end{array}$ \\
\hline CD-22 11432 & 36 days & 1 & 1 & 1.00 & $\ldots$ & $\cdots$ & $\ldots$ & $\cdots$ & $\ldots$ & $\ldots$ & 8 & $(11),(\star)$ \\
\hline GV Tau $S^{t}$ & 38 days & 0.5 & 0.13 & 0.26 & $\cdots$ & $\ldots$ & 55 & 160 & $\ldots$ & $\ldots$ & 0.4 & $(12),(13),(14),(15)$ \\
\hline $\mathrm{KH} 15 \mathrm{D}$ & 48.4 days & 0.715 & 0.74 & 1.03 & 0.29 & 0.57 & $84 \pm 2^{\mathrm{j}}$ & $107 \pm 1^{\mathrm{j}}$ & $92.5 \pm 2.5$ & $\cdots$ & 4 & $\begin{array}{l}\text { (16), (17), (18), (19), } \\
\quad(20),(21),(22)\end{array}$ \\
\hline HD 106906 & 49.2 days & 1.37 & 1.34 & 0.98 & $\ldots$ & 0.67 & 85 & $104^{\mathrm{a}}$ & 88 & $\cdots$ & 15 & $(23),(24)$ \\
\hline YLW $16 A^{t}$ & 92.6 days & $\cdots$ & $\cdots$ & & $\cdots$ & $\cdots$ & $\cdots$ & $\cdots$ & $\cdots$ & $\cdots$ & 1 & $(25)$ \\
\hline AS $205 S^{t}$ & ... & 0.74 & 0.54 & 0.73 & $\cdots$ & $\cdots$ & $66 \pm 2$ & $110 \pm 2$ & $\ldots$ & $\cdots$ & 0.5 & (26), (11), (27) \\
\hline $\mathrm{WL} 4^{\mathrm{t}}$ & 130.87 days & $\cdots$ & $\cdots$ & & $\ldots$ & $\ldots$ & $\ldots$ & $\ldots$ & $\ldots$ & $\cdots$ & 1 & $(28),(29)$ \\
\hline WSB 74 & $<150$ days & 0.86 & 0.817 & 0.95 & $<0.6$ & $\cdots$ & $\cdots$ & $\cdots$ & $\cdots$ & $\cdots$ & 0.1 & $(30),(31),(9)$ \\
\hline WSB 40 & $\cdots$ & 0.96 & 0.75 & 0.78 & 2.3 & $\cdots$ & $\cdots$ & $167 \pm 32^{\mathrm{a}}$ & $\cdots$ & $\cdots$ & 1.5 & $(31),(9)$ \\
\hline V935 Sco & $\cdots$ & 1.11 & 0.75 & 0.68 & 2.6 & $\cdots$ & $\cdots$ & $81 \pm 5^{\mathrm{a}}$ & & & 2.1 & $(31),(9)$ \\
\hline $\begin{array}{l}\text { V853 } \\
\text { Oph Aa-Ab }\end{array}$ & $\ldots$ & $\ldots$ & $\ldots$ & & 3.2 & $\cdots$ & $\cdots$ & $90 \pm 27^{\mathrm{a}}$ & $\ldots$ & $\ldots$ & 2 & $(28),(9),(10)$ \\
\hline ROph 36 & $\ldots$ & 0.73 & 0.61 & 0.84 & 3.3 & $\ldots$ & $\ldots$ & $77 \pm 15^{\mathrm{a}}$ & $\cdots$ & $\cdots$ & 2 & $(32),(33),(31),(9)$ \\
\hline CS Cha & $7 \mathrm{yr}$ & $\cdots$ & $\cdots$ & & 4.0 & $\cdots$ & $24 \pm 3^{\mathrm{a}}$ & $75 \pm 2^{a}$ & $\cdots$ & $\cdots$ & 2 & $(34),(35),(36),(37)$ \\
\hline V892 Tau & $14 \mathrm{yr}$ & 2.25 & 2.25 & 1.00 & 7 & 0.12 & $>60$ & $49 \pm 1^{\mathrm{a}}$ & $60 \pm 4$ & $28 \pm 5^{\mathrm{a}}$ & $<3$ & $(38),(39)$ \\
\hline MHO $2 \mathrm{AB}$ & $\cdots$ & 0.33 & 0.11 & 0.34 & 7.3 & $\cdots$ & $\cdots$ & $\cdots$ & $\cdots$ & $\cdots$ & 3 & (40), (41) \\
\hline $\mathrm{CoKu} \mathrm{Tau} / 4$ & $\cdots$ & 0.5 & 0.5 & 1.00 & 8 & $\cdots$ & $\cdots$ & $\cdots$ & $\cdots$ & $\cdots$ & 4 & $(42),(43),(44)$ \\
\hline IC 348 LRL 31 & $\cdots$ & 1.62 & 0.2 & 0.12 & 8.4 & $\ldots$ & $\ldots$ & $\ldots$ & $\ldots$ & $\ldots$ & 8 & (31), (45) \\
\hline VLA $1623 A^{t}$ & $\ldots$ & $\ldots$ & $\ldots$ & & 30 & $\ldots$ & $\ldots$ & $20^{\mathrm{a}}$ & $\ldots$ & $\ldots$ & $<0.1$ & (46) \\
\hline L1551 IRS 5 & $\cdots$ & 0.65 & $\cdots$ & & 70 & $\cdots$ & 62 & 167 & $\cdots$ & $\cdots$ & 0.1 & (47) \\
\hline L1448 IRS3B ${ }^{t}$ & $\ldots$ & $\ldots$ & $\cdots$ & & 72 & $\ldots$ & 45 & 30 & $\ldots$ & $\ldots$ & $<1$ & (48) \\
\hline HH 250 & $\cdots$ & $\cdots$ & $\cdots$ & & 120 & $\cdots$ & $\cdots$ & $\cdots$ & $\cdots$ & $\cdots$ & $<1$ & (49) \\
\hline $\begin{array}{l}\text { IRAS17216- } \\
\quad 3801\end{array}$ & $\cdots$ & $\cdots$ & $\cdots$ & & 170 & $\cdots$ & $\cdots$ & $\cdots$ & $\cdots$ & $\cdots$ & $<1$ & $(50)$ \\
\hline UY Aur & $1640 \mathrm{yr}$ & $\ldots$ & $\ldots$ & & 190 & $\ldots$ & $50 \pm 10$ & $42 \pm 3$ & $\cdots$ & $\ldots$ & 3 & $(51),(52),(53)$ \\
\hline
\end{tabular}

Notes. Table superscripts are the same as in Table 4. Alternative names: HD $104237=$ DX Cha; ROXs 42C = ROph 26, NTTS 162814-2427; CD$2211432=$ 2MASS J16141107-2305362, GV TauS = Haro6-10N; AS 205S = AS 205B, 2MASS J16113134-1838259; YLW 16A = 2MASS J16272802-2439335; V380 OriA = HH222; W L4 = 2MASS J16271848-2429059, ISO-Oph 128; WSB74 = ROph 32, J16315473-2503238; WSB $40=$ ROph 12; V935 Sco = ROph 2, WSB 12; V853 OphA = SR 13, ROph 23; ROph 36 = 2MASS J16335560-2442049AB; HH $250=$ IRAS 19190+1048; V892 Tau = Elias1.

${ }_{j}^{\text {a }}$ Ambiguity exists in the orbit orientation ( $\mathrm{i}<90$ degrees or $\Omega<90$ degrees) or $(\Omega<180$ degrees or $\Omega>180$ degrees).

$\mathrm{j}$ Inclination and position angle determined from the outflow jet assuming that it is orthogonal to the CB disk. There is a $90^{\circ}$ ambiguity in $i_{\text {disk }}$ and a 180 ambiguity in $\Omega_{\text {disk. }}$.

${ }^{\mathrm{t}}$ Hosts a single companion orbiting beyond the circumbinary disk.

References. (1) Böhm et al. 2004; (2) Garcia et al. 2013; (3) Torres 2004; (4) Monnier et al. 2019; (5) Mathieu et al. 1989; (6) Ghez et al. 1993; (7) Lee et al. 1994; (8) Barsony et al. 2003; (9) Cox et al. 2017; (10) Schaefer et al. 2018; (11) Barenfeld et al. 2016; (12) Menard et al. 1993; (13) Doppmann et al. 2008; (14) Guilloteau et al. 2011; (15) Sheehan \& Eisner 2014; (16) Hamilton et al. 2001; (17) Hamilton et al. 2003; (18) Johnson \& Winn 2004; (19) Chiang \& Murray-Clay 2004; (20) Hamilton et al. 2005; (21) Winn et al. 2006; (22) Aronow et al. 2018; (23) Kalas et al. 2015; (24) De Rosa \& Kalas 2019; (25) Plavchan et al. 2013; (26) Eisner et al. 2005; (27) Kurtovic et al. 2018; (28) Ratzka et al. 2005; (29) Plavchan et al. 2008; (30) Kohn et al. 2016; (31) Ruíz-Rodríguez et al. 2016; (32) Cieza et al. 2010; (33) Orellana et al. 2012; (34) Guenther et al. 2007; (35) Espaillat et al. 2011; (36) Dunham et al. 2016; (37) Ginski et al. 2018; (38) Smith et al. 2005; (39) Monnier et al. 2008; (40) Kraus et al. 2011; (41) Harris et al. 2012; (42) D’Alessio et al. 2005; (43) Ireland \& Kraus 2008; (44) Nagel et al. 2010; (45) Ruíz-Rodríguez et al. 2018; (46) Harris et al. 2018; (47) Takakuwa et al. 2017; (48) Tobin et al. 2016; (49) Comerón et al. 2018; (50) Kraus et al. 2017; (51) Close et al. 1998; (52) Hioki et al. 2007; (53) Tang et al. 2014; ( $\star$ G. Torres 2019, private communication.

that Table 5 may serve as a central repository to motivate future CB follow-up observations. Rodriguez et al. (2015) provide an additional 30 spatially unresolved debris disks from the DEBRIS survey (Matthews et al. 2010) that are likely to be circumbinary.

Many of the circumbinary disks reside in hierarchical triple or quadruple systems, where the companions orbit exterior to the CB disk. We denote these with superscripts in the tables. The frequency of tertiary companions to close spectroscopic binaries is known to be a strong function of binary period: $96 \%$ of binaries with periods $P<3$ days have an outer companion, whereas only $34 \%$ of binaries with 12 days $<P<30$ days have a tertiary (Tokovinin et al. 2006); the latter percentage is consistent with the tertiary rate of our sample (Table 3). There are also two circumternary systems, GW Ori and GG Tau A, which have a single disk encircling all three stars.

\subsection{Direct and Indirect Measurements of Mutual Inclination}

If there are complete measurements of the disk and binary orientations $i_{\text {disk }}, \Omega_{\text {disk }}, i_{\star}, \Omega_{\star}$, then the mutual inclination can be calculated directly via Equation (1). The debris disks in Table 3 are sufficiently nearby that complete astrometric observations of the stellar orbit exist (providing $\Omega_{\star}$ ) and the 
mutual inclination can be determined directly and unambiguously. There are only two protoplanetary disks with sufficiently complete astrometric observations and spatially resolved disk observations for which a similarly precise calculation is possible: AK Sco and HD 98800B. One other protoplanetary system, CoRoT 223992193 (hereafter CoRoT 2239), does not have spatially resolved disk observations or astrometric orbits; however, its mutual inclination has been inferred from photodynamical modeling of its light curve and RVs.

There are several spatially resolved CB protoplanetary disks with astrometric observations in Table 4, but insufficient orbital phase coverage leaves moderate (often correlated) uncertainties in $i_{\star}$ and $\Omega_{\star}$. Because the transformation into mutual inclination is nonlinear, these systems have substantial mutual inclination uncertainties. Even so, near-coplanar mutual inclinations (i.e., $\theta \lesssim 10^{\circ}$ ) are ruled out at high significance for many of these systems: GW Ori, HD 142572, SR 24N, and IRS 43.

The three remaining CB systems, V4046 Sgr, DQ Tau, and UZ Tau E, contain double-lined spectroscopic binaries (SB2s) and have precise measurements of $i_{\text {disk }}, \Omega_{\text {disk}}$, and $i_{\star}$, but not $\Omega_{\star}$. Because we measure $i_{\text {disk }} \simeq i_{\star}$ for each of the three disks in this subsample, we might naïvely conclude that their $\theta$ values are small. In the remainder of this section, we use a standard Bayesian analysis to demonstrate why this supposition is incorrect when each system is considered individually. At the same time, our intuition tells us that it would be strange to live in a universe where $\theta$ is broadly distributed and yet find $i_{\text {disk }} \simeq i_{\star}$ for all three systems. In the upcoming Section 3.3, we introduce a hierarchical Bayesian model that considers all of the $\mathrm{CB}$ disks in the subsample to infer a $\theta$ distribution that is indeed narrow.

Biased measurement of $\theta$ for an individual $C B$ disk around an $S B 2 .-$ Let $\kappa=\left\{i_{\text {disk }}, \Omega_{\text {disk }}, i_{\star}\right\}$ and let $\boldsymbol{D}$ denote the measurements of these parameters. Let $\lambda=\left\{\Omega_{\star}\right\}$ represent the parameters we do not measure directly, so that the full vector of parameters is given by $\boldsymbol{\mu}=\{\boldsymbol{\kappa}, \boldsymbol{\lambda}\}$. To obtain the posterior distribution of $\theta$ given $\boldsymbol{D}$, we first derive a posterior distribution of $\boldsymbol{\mu}$. Then, we use Equation (1) to transform samples from this posterior into samples of the posterior distribution $\theta(\boldsymbol{\mu})$. The likelihood is a multivariate normal

$$
\mathcal{L}=\mathcal{N}(\boldsymbol{D} \mid \kappa, \boldsymbol{\Sigma}),
$$

where the measurement uncertainties on $\boldsymbol{D}$ are described by the covariance matrix $\boldsymbol{\Sigma}$.

For an individual disk, we assume a prior distribution on $\boldsymbol{\mu}$ that specifies the disk and the binary orbit as isotropically oriented in 3D space, i.e., the unit angular momentum vector of each orbit has uniform probability of pointing anywhere on the unit sphere. This is

$$
p(\boldsymbol{\mu})=\frac{\sin i_{\mathrm{disk}} \sin i_{\star}}{4},
$$

where the prior densities of $\Omega_{\text {disk }}$ and $\Omega_{\star}$ are uniform $\in[0,2 \pi]$. We combine the likelihood function with the prior distribution to yield an (unnormalized) posterior probability distribution

$$
p(\boldsymbol{\mu} \mid \boldsymbol{D}) \propto p(\boldsymbol{D} \mid \boldsymbol{\kappa}) p(\boldsymbol{\mu}) .
$$

This posterior distribution is sampled using an MCMC algorithm to generate samples of $\boldsymbol{\mu}$, which are then transformed into $\theta$. For the parameters of V4046 Sgr listed in Table 3, we obtain the posterior in Figure 7.

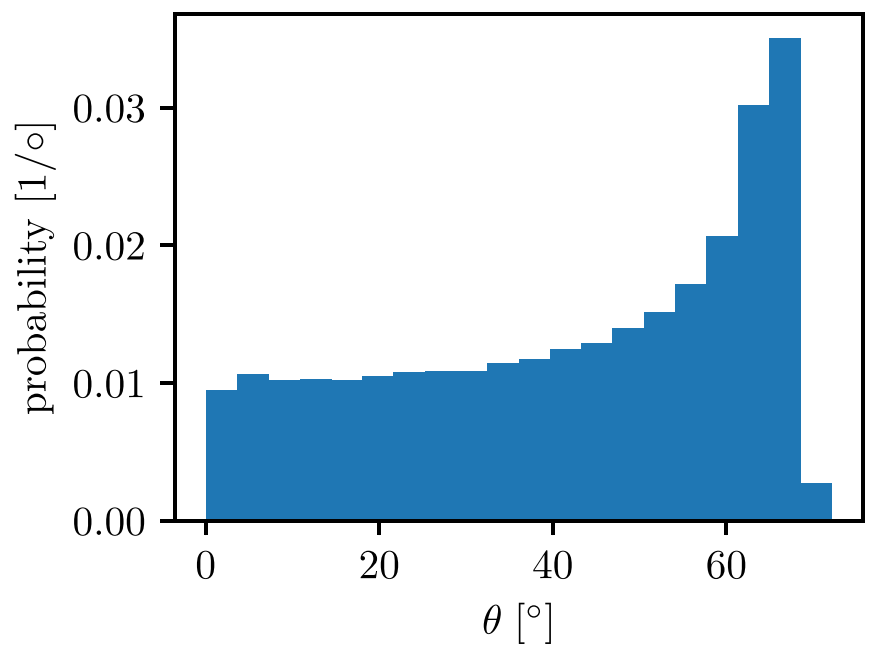

Figure 7. Naïve posterior distribution of $\theta$ for V4046 Sgr, assuming an isotropic prior distribution for the binary. Similar posterior distributions exist for the other CB disks around SB2s in Table 3 when treated on an individual basis.

Even though V4046 Sgr has nearly identical disk and binary inclinations (relative to the sky plane), the posterior distribution has a wide range of permissible $\theta$, telling us that the expected value of $\theta$ is actually much larger than what we would naively assume from $i_{\text {disk }} \simeq i_{\star}$. Without a measurement of $\Omega_{\star}$, the measurement of $i_{\star}$ simply constrains the binary vector to point within a thin annulus on the surface of the unit sphere, the width of which is set by the measurement uncertainty (see Figure 8 for a schematic of such a setup).

While this posterior distribution makes geometrical sense when considering an individual CB system in isolation, when applied to a sample of CB systems that all exhibit $i_{\text {disk }} \simeq i_{\star}$, the statement that $\theta$ is broadly distributed runs counter to intuition. Even though the expected value of the mutual inclination for any individual system is large (e.g., Figure 7), we would suspect that $\theta$ is narrowly distributed near zero; otherwise, we would observe many systems with $i_{\text {disk }} \nsucceq i_{\star}$. The following subsection confirms this suspicion in a statistically rigorous way.

\subsection{Inferring the Mutual Inclinations of CB Disks around SB2s with a Hierarchical Bayesian Model}

Using hierarchical Bayesian analysis, we can explicitly build the mutual inclination distribution (or a parameterization thereof) into our model by considering all disks in the subsample together. Hierarchical Bayesian approaches are useful when there is a natural multilevel structure to a data set (see Loredo 2013 and Sharma 2017 for general introductions in the astrophysical context). Notable applications of hierarchical analysis within the stellar and exoplanetary subfield include inferring the eccentricity distribution of exoplanetary orbits (Hogg et al. 2010), the composition distribution of sub-Neptune planets (Wolfgang \& Lopez 2015), and trends in the stellar obliquity distribution (Muñoz \& Perets 2018).

To implement this hierarchical model, we

1. define a flexible parameterization of the mutual inclination distribution $p(\theta)$;

2. simplify the geometrical relationships between $i_{\text {disk }}, i_{\star}$, and $\theta$ by rotating to a frame centered on the disk;

3. build the full posterior distribution for a sample of $N$ disks; 


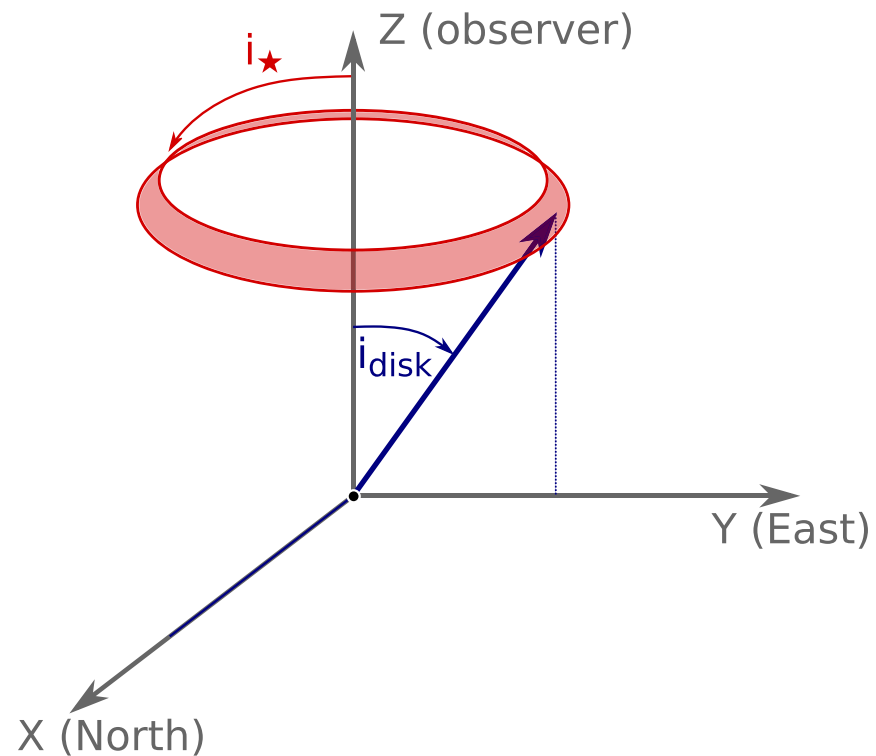

Figure 8. If only the inclination of the binary relative to the sky plane $i_{\star}$ is known (and there is no constraint on $\Omega_{\star}$ ), then the orbit normal of the binary can point anywhere in the red annulus with equal probability. This gives rise to mutual inclination posterior probability distributions like those seen in Figure 7.

4. demonstrate the flexibility of the model by using it to correctly infer the mutual inclination distributions of two very different mock samples of disks; and

5. apply the framework to the real subsample of CB disks around SB2s.

Parameterizing the mutual inclination distribution.-The mutual inclination distribution (at the top level of the hierarchy) can be thought of as a prior $p(\theta)$ on the mutual inclination value $\theta_{j}$ for an individual disk $j$ (at the bottom level of the hierarchy). For example, if $p(\theta)$ favored low mutual inclinations, then for a given disk with $i_{\text {disk }} \simeq i_{\star}$, the posterior distribution $p\left(\theta_{j} \mid \boldsymbol{D}_{j}\right)$ would also favor a low mutual inclination (we would also find $\Omega_{\text {disk }} \simeq \Omega_{\star}$ ). However, if $p(\theta)$ favored a broad range of mutual inclinations, then we might end up with a posterior distribution $p\left(\theta_{j} \mid \boldsymbol{D}_{j}\right)$ more similar to Figure 7.

To simplify the inference process, we assume a functional form for $p(\theta)$. A necessary quality of a mutual inclination distribution is that it is defined over the range $\theta \in(0, \pi)$ and obeys $\lim _{\theta \rightarrow 0} p(\theta)=0$ and $\lim _{\theta \rightarrow \pi} p(\theta)=0$, since exactly aligned and anti-aligned vectors constitute a set of zero measure. There are many classes of functions that could be used-if the data are sufficiently constraining, then the exact choice of functional form will not matter, so long as the function is sufficiently flexible to assume the morphology of the actual mutual inclination distribution. After experimenting with various functional forms, we chose the logit-normal, which provides a wide range of shapes covering the extremes of what we might imagine the mutual inclination distribution to be (i.e., favoring aligned, anti-aligned, or isotropic orientations), while permitting smooth transitions between them. To implement this distribution, we first use the "logit" transformation (e.g., Gelman et al. 2014) to convert $\theta$ from a bounded domain $(0, \pi)$ to an intermediate variable $v$ on an unbounded domain $(-\infty, \infty)$,

$$
v=\operatorname{logit}(\theta / \pi)=\ln \left(\frac{\theta / \pi}{1-\theta / \pi}\right) .
$$

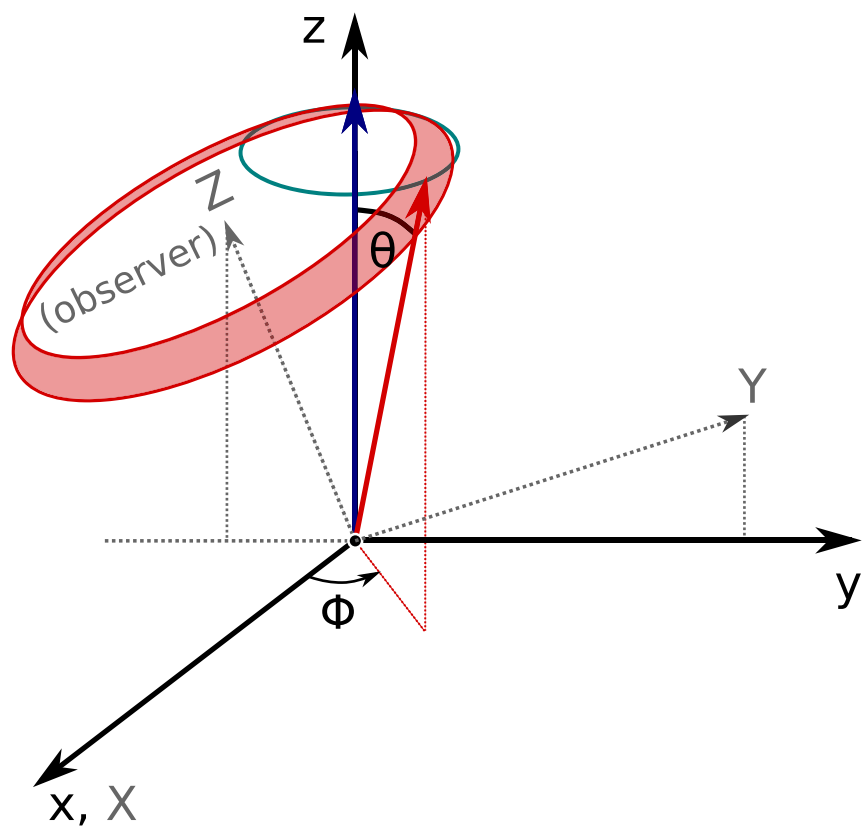

Figure 9. The $x y z$ coordinate system in the frame of the circumbinary disk, where the $z$-axis is aligned with the disk unit angular momentum vector. As before, the blue and red vectors are the orbit normals of the disk and binary, respectively. For a given $\theta$, the red annulus specifies the constraint on constant $i_{\star}$ as in Figure 8. The teal circle represents a constant value of $\theta$.

For each system $j$, we say that $v_{j}$ is drawn from the distribution

$$
v_{j} \sim \mathcal{N}\left(\mu_{v}, \tau_{v}\right)
$$

which is a normal distribution with mean $\mu_{v}$ and precision $\tau_{v}=1 / \sigma_{v}^{2}$, where $\sigma_{v}$ is the standard deviation of the normal. Together, we call these the hyperparameters of the mutual inclination distribution $\boldsymbol{\alpha}=\left\{\mu_{v}, \tau_{v}\right\}$. The normal distribution on $v_{j}$ is equivalent to a prior probability distribution on $\theta_{j}$ of

$$
\begin{aligned}
p\left(\theta_{j} \mid \boldsymbol{\alpha}\right)= & \frac{1}{\theta(1-\theta / \pi)} \sqrt{\frac{\tau_{v}}{2 \pi}} \\
& \times \exp \left(-\frac{\tau_{v}}{2}\left(\operatorname{logit}(\theta / \pi)-\mu_{v}\right)^{2}\right) .
\end{aligned}
$$

We also experimented with different functional forms for $p(\theta \mid \boldsymbol{\alpha})$, including reparameterized Beta functions, and found our results to be unchanged.

Rotating into the frame of the disk.-In Figure 9, we introduce a new $x y z$ coordinate system in the frame of the circumbinary disk, which simplifies the mathematical relationships between $\theta$ and the disk and binary orbit normals. In this frame, the disk angular momentum unit vector is aligned with the $z$-axis, $\theta$ is the polar angle of the binary unit angular momentum vector, and $\phi$ denotes the azimuthal angle of the binary vector. The relationship between the observer frame $X Y Z$ (Figures 1 and 8) and the disk frame $x y z$ (Figure 9) is defined by two rotations about the $Z$ - and $x$-axes by the angles $\Omega_{\text {disk }}$ and $i_{\text {disk }}$, respectively. Because $\Omega_{\star}$ is unmeasured, the specific value of $\Omega_{\text {disk }}$ is irrelevant to the calculation, and so we set $\Omega_{\text {disk }}=0$ to simplify the required operations (as in Figure 8), which also means $X=x$. Then, all we require is 
the rotation matrix

$$
\boldsymbol{P}_{x}\left(i_{\text {disk }}\right)=\left[\begin{array}{ccc}
1 & 0 & 0 \\
0 & \cos i_{\text {disk }} & -\sin i_{\text {disk }} \\
0 & \sin i_{\text {disk }} & \cos i_{\text {disk }}
\end{array}\right],
$$

which is defined such that its application results in a clockwise rotation of the axes as viewed from $+x$-axis.

For a given $\theta$ and $\phi$, the location of the binary vector in the disk frame (Figure 9) is

$$
\left[\begin{array}{l}
x \\
y \\
z
\end{array}\right]=\left[\begin{array}{c}
\sin \theta \cos \phi \\
\sin \theta \sin \phi \\
\cos \theta
\end{array}\right] .
$$

For a specific value of $\theta$, then, the location of the binary vector is constrained to a ring at a constant angular distance from the $z$-axis (teal circle, Figure 9). The most probable locations along the ring (given by $\phi$ ) are those that coincide with the measurement of $i_{\star}$, which is represented by the inclined red annulus in Figure 9. To calculate $i_{\star}$ from $(\theta, \phi)$ along the ring requires the inverse of the rotation matrix

$$
\left[\begin{array}{c}
X \\
Y \\
Z
\end{array}\right]=\boldsymbol{P}_{x}^{-1}\left(i_{\mathrm{disk}}\right)\left[\begin{array}{l}
x \\
y \\
z
\end{array}\right],
$$

which is simply the transpose of $\boldsymbol{P}_{x}$. Then, $i_{\star}$ is the angle between the $Z$-axis and the binary orbit normal, so that

$$
\cos i_{\star}=\cos i_{\text {disk }} \cos \theta-\sin i_{\text {disk }} \sin \theta \sin \phi .
$$

The main benefit of working in the disk frame is that the prior on the binary orientation is separable,

$$
p\left(\theta_{j}, \phi_{j}\right)=p\left(\theta_{j}\right) p\left(\phi_{j}\right),
$$

where $p(\theta)$ is specified by Equation (7) and $p(\phi)$ is uniform $\in\left[\begin{array}{ll}0,2 \pi\end{array}\right]$. Equation (11) defines a relationship between these parameters and $\cos i_{\star}$, enabling us to write

$$
\begin{aligned}
& p\left(\cos i_{\star} \mid \cos i_{\text {disk }}, \theta, \phi\right) \\
& \quad=\delta\left(\cos i_{\star}-\left(\cos i_{\text {disk }} \cos \theta-\sin i_{\text {disk }} \sin \theta \sin \phi\right)\right) .
\end{aligned}
$$

Technically there are two values of $\phi \in[0,2 \pi]$ that yield the same $\cos i_{\star}$. Because of the symmetry of the annulus and ring across the $y-z$ plane, we can make Equation (13) a one-to-one relationship by limiting the range to $\phi \in[-\pi / 2, \pi / 2]$ without loss of generality.

The full posterior distribution for the CB subsample.-Let $\kappa_{j}=$ $\left\{\cos i_{\text {disk }, j}, \cos i_{\star, j}\right\}, \quad D_{j}=\left\{\cos i_{\text {disk }, j}, \cos i_{\star, j}\right\}, \quad \lambda_{j}=\left\{\theta_{j}, \phi_{j}\right\}$, and $\boldsymbol{\mu}_{j}=\left\{\boldsymbol{\kappa}_{j}, \boldsymbol{\lambda}_{j}\right\}$. The posterior probability distribution for the parameters of an individual disk is

$$
p_{j}\left(\boldsymbol{\mu}_{j} \mid \boldsymbol{D}_{j}, \boldsymbol{\alpha}\right) \propto \mathcal{N}\left(\boldsymbol{D}_{j} \mid \boldsymbol{\kappa}_{j}, \boldsymbol{\Sigma}_{j}\right) p\left(\boldsymbol{\mu}_{j} \mid \boldsymbol{\alpha}\right) .
$$

As before, the likelihood function is evaluated with the observed parameters, while the parameters in $\boldsymbol{\lambda}_{j}$ are constrained by the prior from the mutual inclination distribution and the functional relationship between $\theta, \phi$, and $\cos i_{\star}$. The prior is

$$
\begin{aligned}
p\left(\boldsymbol{\mu}_{j} \mid \boldsymbol{\alpha}\right)= & p\left(\cos i_{\star, j} \mid \cos i_{\mathrm{disk}, j}, \theta_{j}, \phi_{j}\right) \\
& \times p\left(\cos i_{\mathrm{disk}, j}\right) p\left(\theta_{j} \mid \boldsymbol{\alpha}\right) p\left(\phi_{j}\right),
\end{aligned}
$$

where $p\left(\cos i_{\mathrm{disk}, j}\right)$ is a geometrical prior as before. Note that the posterior distribution for an individual disk is conditional on

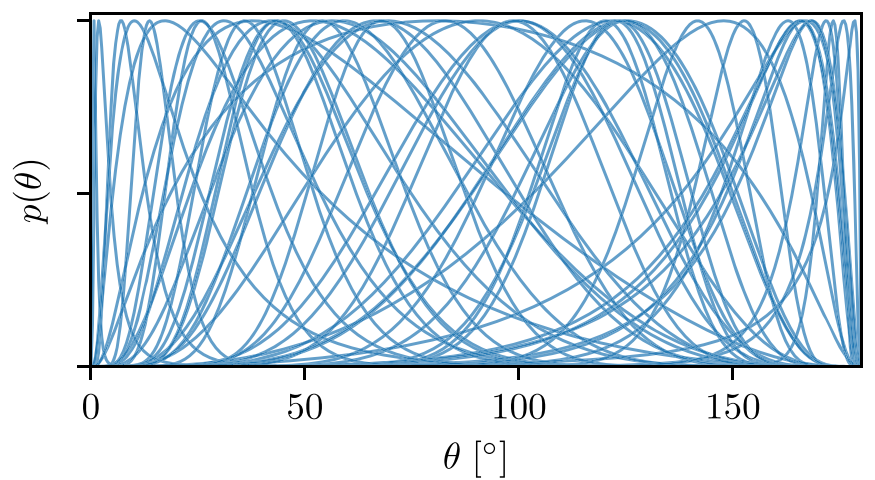

Figure 10. Range of mutual inclination distributions $p(\theta \mid \boldsymbol{\alpha})$ randomly drawn from the hyperpriors $p(\boldsymbol{\alpha} \mid \boldsymbol{\beta})$, for a fixed choice of hyperprior parameters $\boldsymbol{\beta}$. To highlight the range of morphologies allowed, each draw has been scaled such that its peak value is 1 . The range of functional forms demonstrate that the logit-normal distribution can easily mimic distributions that favor mostly aligned, anti-aligned, or isotropic mutual inclinations and easily transition between them.

the values within $\alpha$ (which control whether the mutual inclination distribution favors low, isotropic, or high values of $\theta$ ), reflecting the hierarchical nature of the problem.

The full posterior distribution is given by

$$
p\left(\left\{\boldsymbol{\mu}_{j}\right\}_{j=1}^{N}, \boldsymbol{\alpha} \mid\{\boldsymbol{D}\}_{j=1}^{N}\right)=\prod_{j}^{N} p_{j}\left(\boldsymbol{\mu}_{j} \mid \boldsymbol{D}_{j}, \boldsymbol{\alpha}\right) \times p(\boldsymbol{\alpha} \mid \boldsymbol{\beta}),
$$

where $N$ is the number of systems in the sample. This posterior distribution contains $N \times 3+2$ parameters that need to be sampled - the orientation parameters for each system, plus the two hyperparameters of the mutual inclination distribution in $\boldsymbol{\alpha}$. To form a proper posterior probability distribution, the hyperparameters also require their own hyperpriors $p(\boldsymbol{\alpha} \mid \boldsymbol{\beta})$, where $\boldsymbol{\beta}$ are the settings of the hyperprior distribution. These hyperpriors on $\boldsymbol{\alpha}$ are simply chosen such that the range of functional realizations from the logit-normal distribution covers the range of distributions that we hope to infer, without falling victim to pathologies in implementation. From visual inspection of various functional realizations, we decide on a Gaussian prior on $\mu_{v}$ of $p\left(\mu_{v}\right) \propto$ $\mathcal{N}\left(\mu_{v} \mid \mu_{\mu}=0, \sigma_{\mu}=2\right)$. While the precision parameter $\tau_{v}$ is already defined to be positive, we find that for very low values $(\tau<0.5)$ the mutual inclination distribution $p(\theta \mid \boldsymbol{\alpha})$ becomes multimodal with peaks near 0 and $\pi$. To avoid this behavior, we enforce a half-Gaussian prior $p\left(\tau_{v}\right) \propto \mathcal{N}_{1 / 2}\left(\tau_{v} \mid \mu_{\tau}=0.5, \sigma_{\tau}=4\right)$ where $\tau>0.5$; otherwise, $p\left(\tau_{v}\right)=0$. We refer to this collection of hyperprior parameters as $\boldsymbol{\beta}=\left\{\mu_{\mu}, \sigma_{\mu}, \mu_{\tau}, \sigma_{\tau}\right\}$, and their values remain fixed throughout the entire inference process. To demonstrate the range of possible mutual inclination distributions under this choice of prior, we show random samples from the hyperprior in Figure 10.

In Figure 11 we show the probabilistic graphical model representing this posterior distribution (Equation (16)). Each node in the graph represents a random variable with a probability distribution attached to it. Arrows between nodes represent causal relationships between parameters. The box or "plate" represents the $N$ systems in our sample, and so there are individual nodes for each system for all variables within the plate.

Testing the model with two mock $C B$ disk samples.-To demonstrate the flexibility of this hierarchical Bayesian model, 


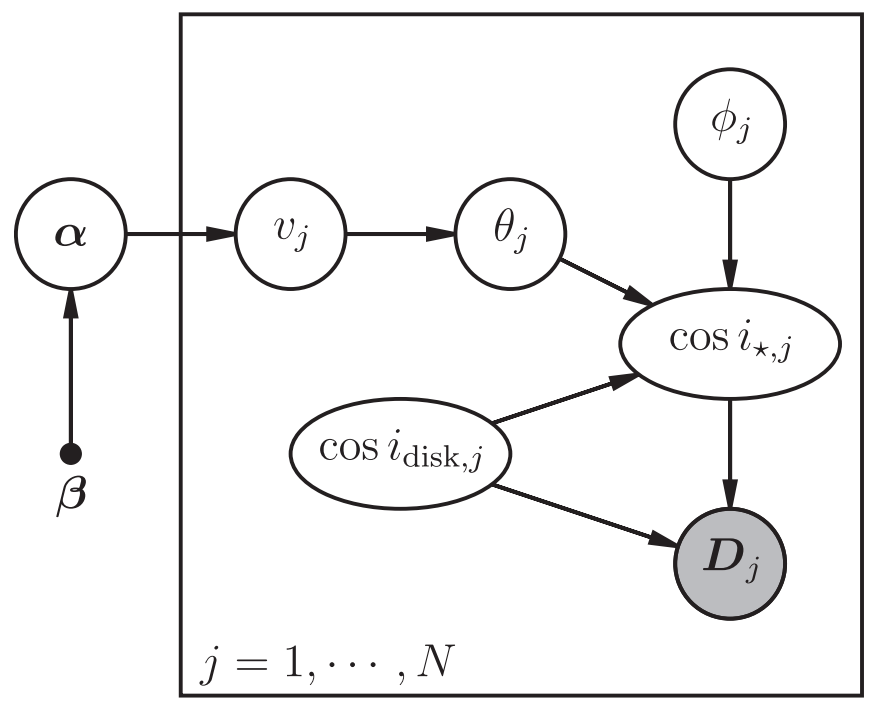

Figure 11. Probabilistic graphical model representing the causal relationships between the parameters in our hierarchical Bayesian model. The hyperprior parameters $\boldsymbol{\beta}$ (fixed) describe the range of possible mutual inclination distributions for all disks in the sample (parameterized by $\boldsymbol{\alpha}$, Equation (7)). Within the plate are parameters representing each individual system $j$. The intermediate mutual inclination variable $v_{j}$ is drawn from the mutual inclination distribution and converted to $\theta_{j}=\pi \operatorname{logit}^{-1}\left(v_{j}\right)$. Then, $\cos i_{\text {disk }, j}$ and $\phi_{j}$ are drawn from their prior distributions, and Equation (13) is used to calculate $\cos i_{\star, j}$. Finally, the likelihood for each system is evaluated via the agreement of $\kappa_{j}=\left\{\cos i_{\text {disk }, j}, \cos i_{\star, j}\right\}$ with the observed values in $\boldsymbol{D}_{j}$.

we apply it to two $N=10$ samples of fake circumbinary disk systems with drastically different mutual inclination distributions. The first sample contains disks and binaries with isotropic orientations, i.e., $p_{\text {iso }}(\theta) \propto \sin (\theta)$. The second sample utilizes the same disk orientations, but the binary orientations have been drawn from a mutual inclination distribution favoring aligned orientations, $p_{\text {low }}(\theta)=\mathcal{N}\left(\theta \mid \mu_{\theta}, \sigma_{\theta}\right) \sin \theta$, which is a Gaussian with $\mu_{\theta}=5^{\circ}$ and $\sigma_{\theta}=2^{\circ}$, tapered by a $\sin (\theta)$ profile to satisfy the limit condition. We generate $\theta$ samples from this distribution using rejection sampling (see Mackay 2003, Chap. 29.3), draw samples of $\phi$ uniform in the range $[0,2 \pi]$, and convert these $\theta, \phi$ samples to $\cos i_{\star}$ using Equation (13). We assume a $1^{\circ}$ uncertainty on $i_{\text {disk }}$ and $i_{\star}$ measurements. Inspecting these two samples of fake systems, our intuition from earlier is confirmed by the fact that the majority of disks in the isotropic sample have $i_{\text {disk }} \nsucceq i_{\star}$ while all disks in the low mutual inclination sample have $i_{\text {disk }} \simeq i_{\star}$.

For a sample of 10 systems our hierarchical model requires 32 parameters. This high-dimensional parameter space is common to hierarchical problems and is challenging to explore using MCMC algorithms popular with astronomers, such as the Metropolis-Hastings algorithm and the affine-invariant ensemble sampler (Goodman \& Weare 2010; Foreman-Mackey et al. 2013). One approach to hierarchical sampling is the $K$-samples method (as described in Hogg et al. 2010), whereby independent samples from (lower-dimensional) individual disk posteriors are reweighted under the hierarchical prior to approximately calculate $p(\boldsymbol{\alpha} \mid \boldsymbol{D})$. This approach is useful when the modeler has access to samples of the likelihood for each system but not the individual data sets required to calculate the likelihood directly. Because we have access to the data of the individual systems themselves (in Table 3), we choose to directly sample the full high-dimensional posterior with a version of the Hamiltonian Monte Carlo (HMC) algorithm called the No U-Turn Sampler (Hoffman \& Gelman 2014), implemented in PyMC3 (Salvatier et al. 2016).

In addition to evaluating the posterior density at each sample, HMC algorithms also calculate the gradient of the posterior with respect to the model parameters in order to simulate the evolution of a dynamic system. The gradient is calculated to machine precision using automatic differentiation as provided by $\mathrm{PyMC} 3$ through the Theano framework (Theano Development Team 2016). The use of gradient information makes HMC samplers very efficient in higherdimensionality spaces and effective at exploring the highly correlated pathologies common to hierarchical models (Betancourt \& Girolami 2013; Betancourt 2017). Via the MCMC sampling process, we obtain joint samples of $\left\{\boldsymbol{\mu}_{j}\right\}_{j=1}^{N}$ from the full posterior distribution. It is straightforward to obtain samples of the $\left\{\theta_{j}\right\}_{j=1}^{N}$ and $\boldsymbol{\alpha}$ marginalized over the other dimensions by simply dropping these other dimensions from the multivariate output chain.

We apply our hierarchical Bayesian model separately to each of the two samples using only the information in $\boldsymbol{D}=$ $\left\{\cos i_{\text {disk }, j}, \cos i_{\star, j}\right\}_{j=1}^{N}$ and show marginal posteriors of the mutual inclinations in Figure 12 (top half is the "iso" sample, bottom half is the "low" sample). In the wide subpanels we show realizations of the mutual inclination distribution $p(\theta \mid \boldsymbol{D}, \boldsymbol{\alpha})$ generated from draws from the marginal posterior of $p(\boldsymbol{\alpha} \mid \boldsymbol{D})$. We represent the mutual inclination distribution that generated each fake data set with a black curve. In both instances, we infer mutual inclination distributions that closely hew to the true distribution. We deliberately chose fake distributions $p_{\text {iso }}$ and $p_{\text {low }}$ that were not explicit subsets of our mutual inclination parameterization to demonstrate that $p(\theta \mid \boldsymbol{\alpha})$ has sufficient flexibility to accurately model these distributions, regardless. The remainder of the panels in Figure 12 show the marginal posteriors of $p\left(\theta_{j} \mid \boldsymbol{D}\right)$ for the individual disks in each of the samples. We mark the true mutual inclination for each system with a black line. For the isotropic sample, although the inferences of the individual mutual inclinations are broad, the posterior does bracket the true $\theta_{j}$ in every case. For the low mutual inclination sample, we see that the mutual inclination of each system is inferred to be low. These drastically different distributions were recovered using the same hierarchical model implementation (and choice of hyperpriors) with access to only the values of $i_{\text {disk }}$ and $i_{\star}$ in each sample, demonstrating that the hierarchical model has the power to accurately discriminate between a range of generating mutual inclination distributions.

Inferring the mutual inclinations of the subsample of $C B$ disks around SB2s.-Finally, we apply our hierarchical Bayesian model to the subsample of CB disks around SB2s: V4046 Sgr, DQ Tau, UZ Tau E, and AK Sco. Even though there is an astrometric orbit for the AK Sco binary (Anthonioz et al. 2015), the model used for the interferometric fit included a narrow ring with radius $\sim 0.5 \mathrm{au}$ to mimic the contribution from the inner edge of a circumbinary disk. Subsequent scattered light observations (Dong et al. 2016; Janson et al. 2016) and submillimeter observations (I. Czekala et al. 2019, in preparation) revealed that the dust in AK Sco is actually distributed in a narrow ring with a radius of $\sim 30 \mathrm{au}$, raising the possibility that the stellar orbital parameters from the interferometric model could be biased. For the purposes of the hierarchical model, we ignore the AK Sco astrometric orbit and treat $\Omega_{\star}$ and $\theta$ as unknown.

We present the mutual inclination distribution inferred from this subsample in Figure 13. In addition to plotting 20 random 


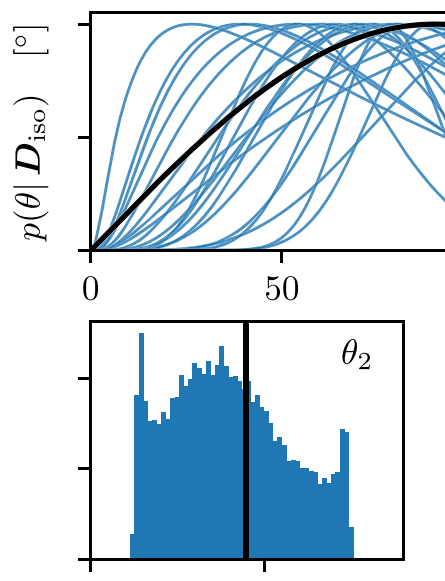

0

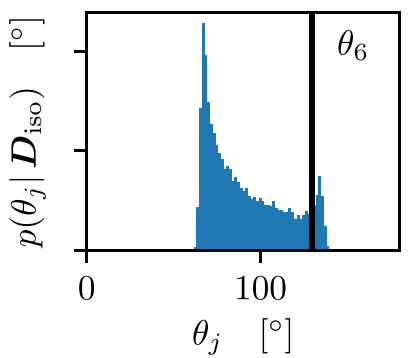

$\left.\theta_{j} \quad{ }^{\circ}\right]$

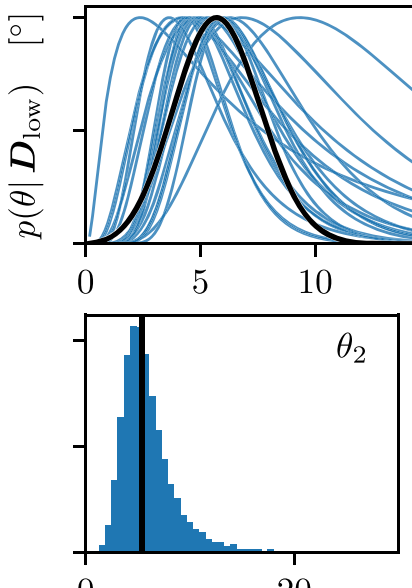

0

20

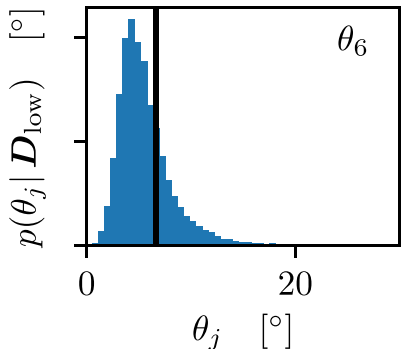

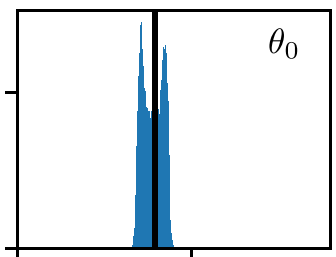

$100 \quad 150$

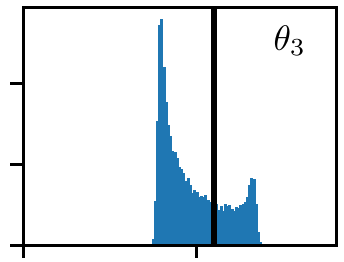

$0 \quad 100$

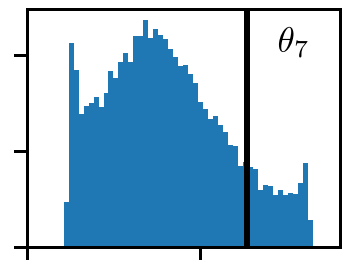

$0 \quad 100$
0

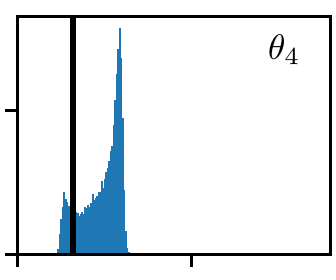

100

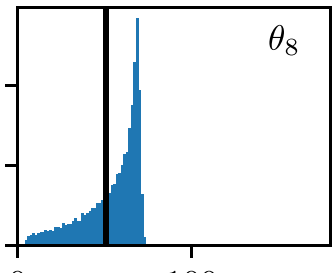

100

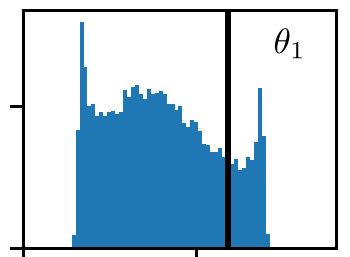

$0 \quad 100$

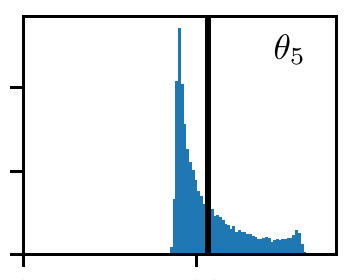

$0 \quad 100$

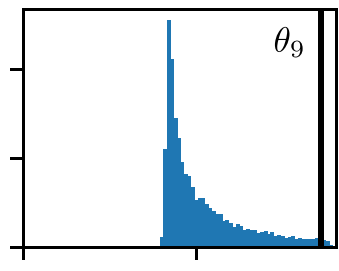

$0 \quad 100$

isotropic sample

low $\theta$ sample
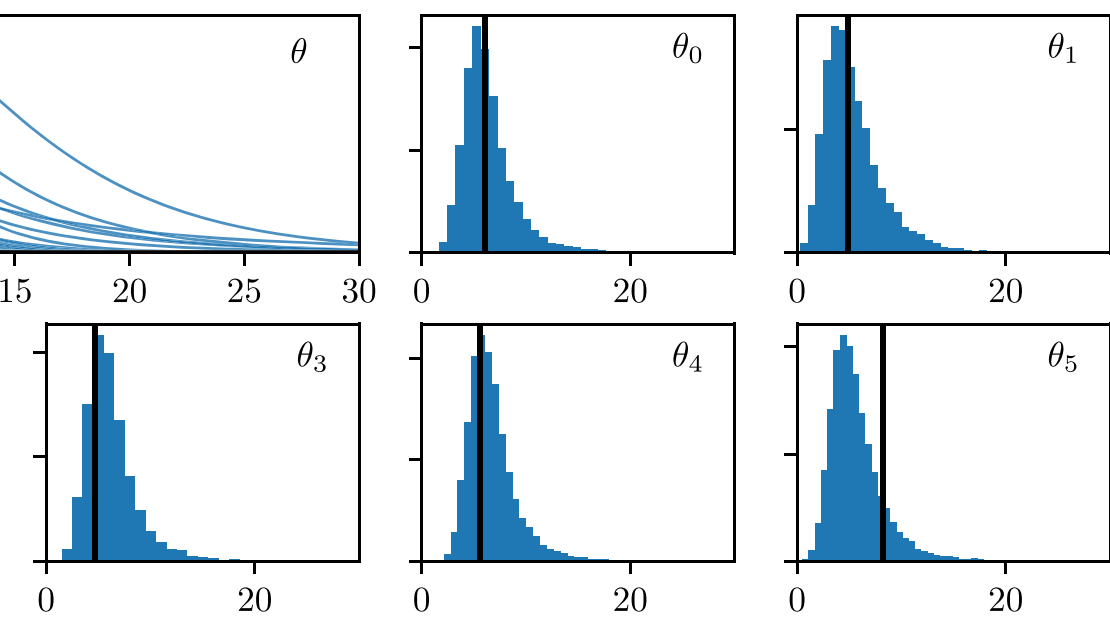

$0 \quad 20 \quad 0$

20
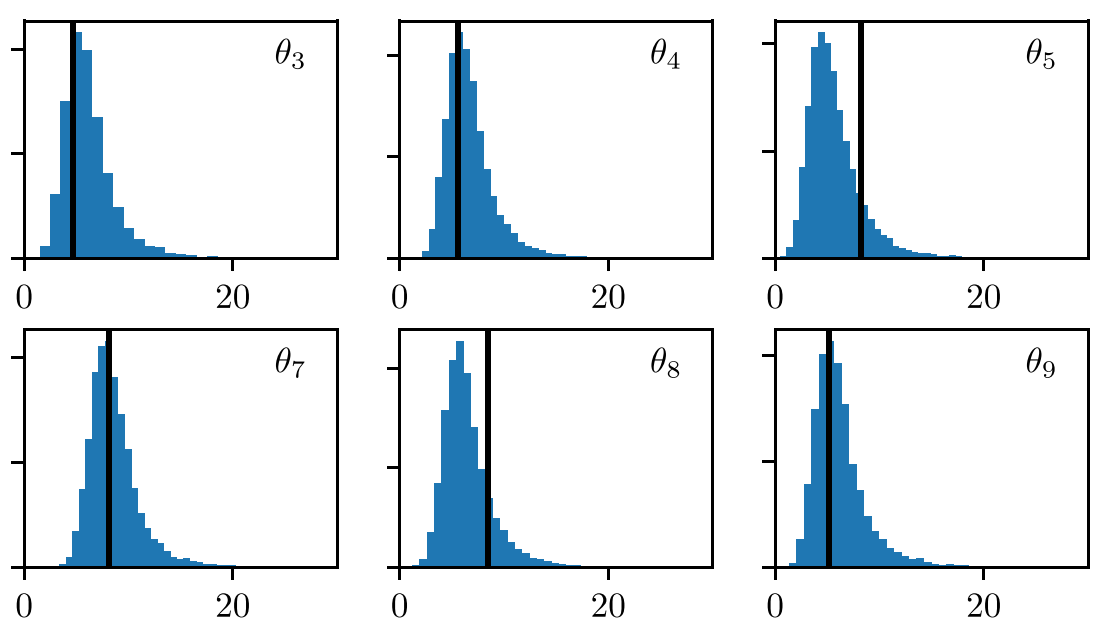

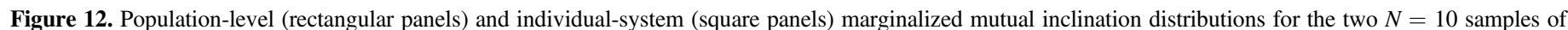

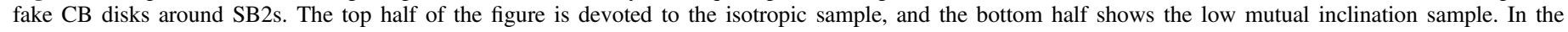

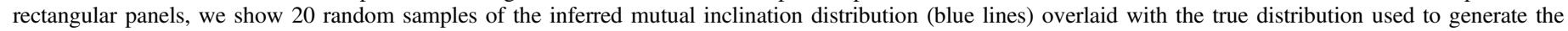

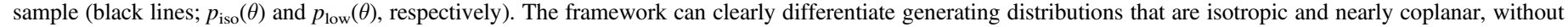

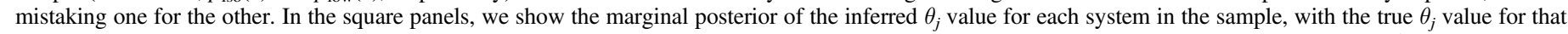

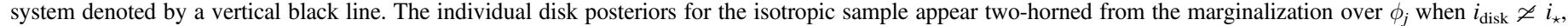

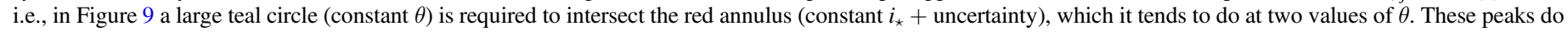

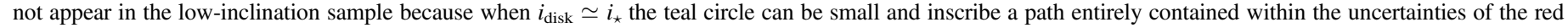
annulus. 

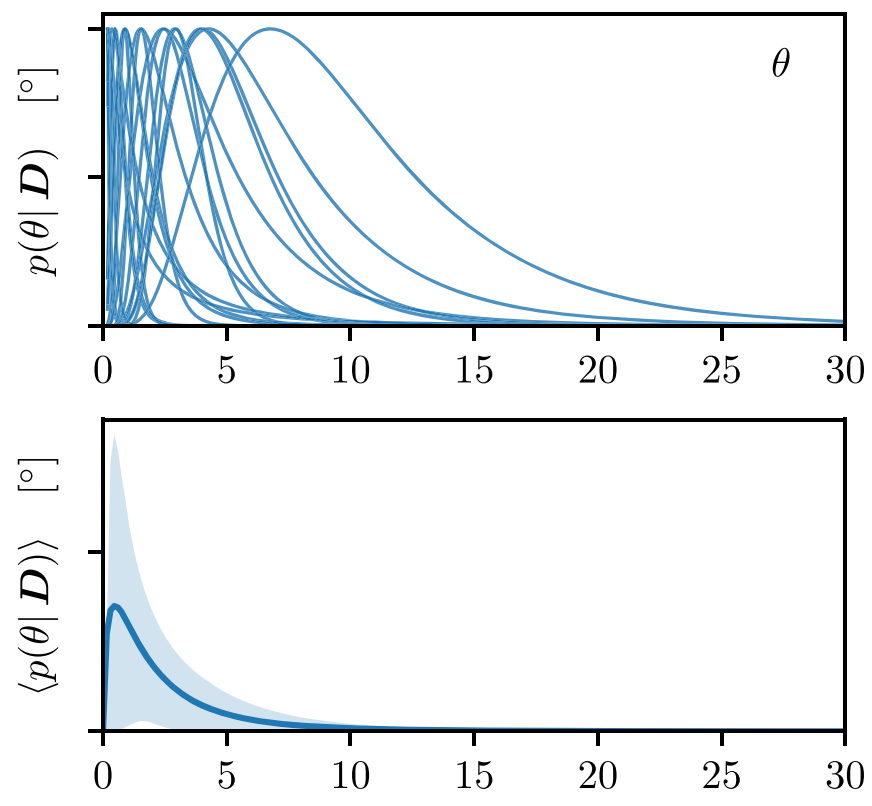

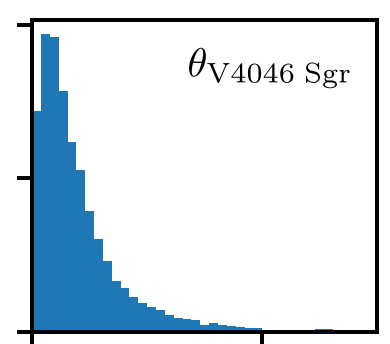

0

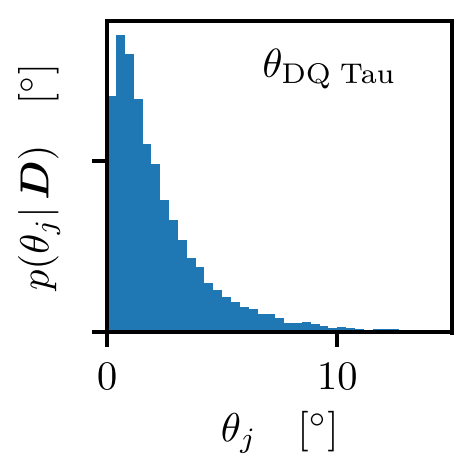

Figure 13. Same as Figure 12, but utilizing the subsample of protoplanetary disks around spectroscopic binaries: V4046 Sgr, AK Sco, DQ Tau, and UZ Tau E. In the second panel, we show the mean value of $p(\theta \mid \boldsymbol{D})$ with a $68 \%$ confidence interval calculated from all of the draws.

draws of the mutual inclination distribution, in the second panel we also represent $p(\theta \mid \boldsymbol{D})$ in an alternate form by showing the mean value of the $p(\theta \mid \boldsymbol{D})$ draws with a shaded $68 \%$ confidence interval. These two representations are equivalent visualizations of the uncertainty in the inferred mutual inclination distribution. The distribution clearly favors low mutual inclinations, with $68 \%$ of the probability contained within $\theta<3.0 \quad(95.4 \%$ and $99.7 \%$ within $\theta<9.5$ and $\theta<11^{\circ} .9$, respectively). We summarize the marginal $\theta_{j}$ posteriors of the individual disks in Table 3.

\subsection{Mutual Inclination as a Function of Orbital Period}

We now have a sample of 19 binary orbits with circumbinary (or circumternary) disks with mutual inclination measurements
(Tables 3 and 4). We plot these systems, along with the Kepler circumbinary planets, as a function of period, semimajor axis, and eccentricity in Figure 14. We find that of the six protoplanetary $\mathrm{CB}$ disks orbiting stars with $P<40$ days, five of them have mutual inclinations constrained to $\theta<5^{\circ}$; the sixth (TWA 3A) is consistent with this sample, though with a larger uncertainty on $\theta$. All three of the debris disks in this period range are also consistent with having low mutual inclinations, although their upper limits on $\theta$ are also not as stringent $\left(\theta \lesssim 25^{\circ}\right)$. At these shorter binary periods, the distribution of $\mathrm{CB}$ disk mutual inclinations is consistent with that of the Kepler CB planets, which all have $\theta<5^{\circ}$ and orbit binaries with $P \leqslant 41$ days.

For the $10 \mathrm{CB}$ disks orbiting binaries with periods longer than 40 days, the distribution of mutual inclinations differs dramatically, with most systems having values of $\theta>20^{\circ}$. Although the sample size is limited, it appears as though there are two clusters of systems, substantially misaligned systems with $\theta \sim 40^{\circ}$ and drastically misaligned systems with nearpolar disk orientations $\theta \sim 90^{\circ}$. Only one disk (HD 200775) has a mutual inclination $\theta<20^{\circ}$. There is a significant trend that misaligned disks surround only the most eccentric binaries ( $e \gtrsim 0.3$; see the last paragraph of Section 4.1 for a discussion of the point at $(e, \theta)=\left(0.1,45^{\circ}\right)$ corresponding to the GW Ori circumternary disk).

It is unlikely that these trends result from observational selection effects. The probability of detecting a disk from its spatially unresolved thermal radiation is independent of disk inclination, provided that radiation originates from material that is optically thin (which debris disks are at all wavelengths, and protoplanetary disks may be in the submillimeter). In addition, we know of no observational bias that would correlate (in whatever sense) the orientations of binary orbits with those of their surrounding disks. For example, while RV searches for spectroscopic binaries are more sensitive to edge-on orbits, and while debris disks may be more easily spatially resolved when viewed edge-on owing to higher line-of-sight column densities, there is no bias that would correlate their relative nodal orientations on the sky.

\section{Discussion}

\subsection{Disk-Binary Interactions}

Turbulent fragmentation of a collapsing molecular core (Offner et al. 2010) and fragmentation of a protostellar disk by gravitational instability (Kratter et al. 2008) are two ways by which stellar binaries can directly form. However, neither of these mechanisms appears capable of forming protostars with initial separations $a<10$ au. Direct fragmentation on small scales requires high densities, which renders gas optically thick, supported by thermal pressure, and resistant to cooling and collapse (Larson 1969; Bate et al. 2002).

Close binaries are likely made instead from initially wide binaries that underwent subsequent hardening (e.g., Bate 2019, and references therein). A variety of mechanisms exist to shrink binary orbits: dynamical interactions with third parties (via, e.g., Lidov-Kozai oscillations and tidal friction), dissipative interactions with circumstellar and circum-multiple disks, and the accretion of low angular momentum gas onto the binary from a residual core/envelope (Artymowicz \& Lubow 1996; Bate et al. 2002; Bate 2012). Moe \& Kratter (2018) found that Lidov-Kozai cycles are insufficient to explain the full 


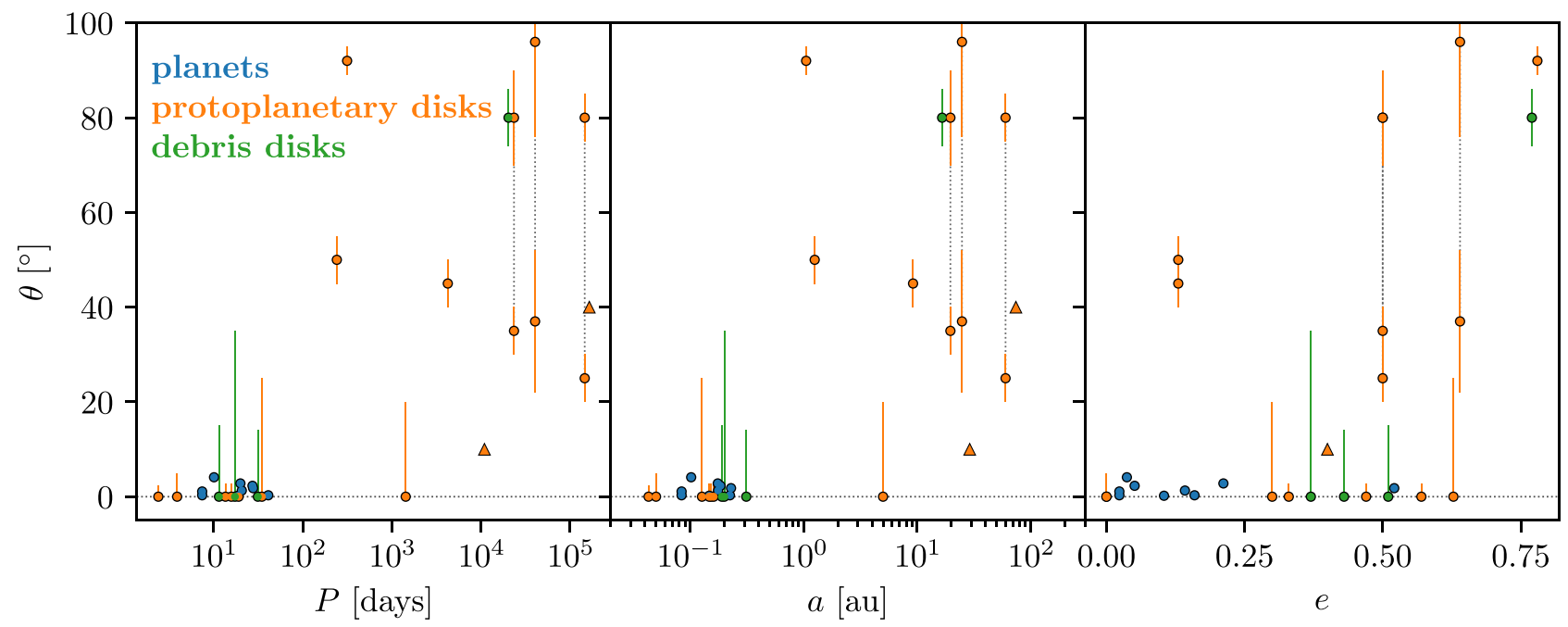

Figure 14. Left: mutual inclinations of Kepler circumbinary planets and all circumbinary protoplanetary and debris disks in Tables 3 and 4 , as a function of binary orbital period. The triangles represent the lower limits on $\theta$ for R CrA and IRS 43. The dotted lines connect degenerate solutions for HD 142527, SR 24N, and GG Tau Aa-Ab. Middle: mutual inclination as a function of semimajor axis. Right: mutual inclination as a function of binary eccentricity. The triangle represents the lower limit on $\theta$ for $\mathrm{R} \mathrm{CrA} \mathrm{(} e$ is unknown for IRS 43, and so it is not plotted in the eccentricity panel). The two points at $e=0.13$ correspond to GW Ori A-B and AB-C, which collectively host a circumternary protoplanetary disk. Long-period, eccentric binaries are more likely to host circumbinary disks with significant mutual inclinations.

population of close binaries: even among those binaries hosting a tertiary star, $60 \%$ require extra dissipative interactions with primordial gas to reach their current separations. In a large radiation-hydrodynamical simulation of a collapsing molecular cloud, Bate (2019) found that a close binary typically forms when two initially unbound stars become bound and shrink their orbit by gravitational interactions mediated by their disks. Gas-rich disks provide both a larger cross section for encounters and a means of dissipating orbital energy.

Binaries that form by dissipative encounters may be orbited by disks having an initially wide range of mutual inclinations (Bate 2018). The inclinations can evolve by subsequent diskbinary interactions. Foucart \& Lai $(2013,2014)$ found that when the binary orbit is circular or nearly so, and when the relative disk-binary inclination is small (but nonzero), gravitational torques between the warped disk and the binary can bring their mean planes into alignment. These authors computed an alignment timescale that is short compared to the disk lifetime unless the viscosity governing inclination damping is much smaller than the viscosity controlling radial diffusion of mass (see also Lodato \& Facchini 2013), or the inner edge of the disk is far removed from the binary.

If, however, the binary is substantially eccentric, it can force the disk out of alignment. The orbit of a circumbinary test particle, if initially sufficiently inclined, librates (oscillates) about $\theta=90^{\circ} ;{ }^{12}$ the more eccentric the binary, the smaller is the initial inclination required to access this libration (Verrier \& Evans 2009; Farago \& Laskar 2010; Naoz et al. 2017; Vinson $\&$ Chiang 2018). These librations are damped in a viscous disk, which seeks to settle into a polar configuration (Martin \& Lubow 2017; Lubow \& Martin 2018; Zanazzi \& Lai 2018) or near-polar configuration if the disk mass is high (Martin \& Lubow 2019). In the parameter survey of Martin \& Lubow (2018), disks initially misaligned by $30^{\circ}$ or more around a binary with $e=0.8$ undergo damped librations about $\theta=90^{\circ}$;

\footnotetext{
${ }^{12}$ The fixed point at $\theta=90^{\circ}$ represents the strongest, quadrupole-order resonance. Other resonances with other fixed points are surveyed by Vinson \& Chiang (2018) in the test particle limit.
}

disks with smaller initial inclinations are not attracted to the polar configuration but still exhibit inclination variations of tens of degrees (see, e.g., their Figure 12).

As documented in Tables 3 and 4, binaries with $P<10$ days are circularized - presumably from tidal dissipation. In support of the theoretical ideas discussed above, Figure 14 attests that CB disks orbiting short-period binaries are coplanar (or nearly so) with their hosts, while CB disks orbiting long-period, eccentric binaries exhibit a variety of mutual inclinations ranging up to $\theta \approx 90^{\circ}$. The polar orientations of HD 98800B and $99 \mathrm{Her}$ are consistent with damped librations about the fixed point of $\theta=90^{\circ}$ in eccentric binaries (Martin \& Lubow 2017); these disks also exhibit the nodal orientations expected from this scenario (e.g., Lubow \& Martin 2018; Zanazzi \& Lai 2018; Kennedy et al. 2019). ${ }^{13}$ Those disks that are not polar or substantially inclined around binaries with eccentricities of $\sim 0.5$ may have formed with inclinations below the threshold required for polar librations (Foucart \& Lai 2014; Martin \& Lubow 2018). A few of these disks orbit binaries with periods of 10-40 days and may have had their inclinations damped while driving their hosts into more compact configurations. Notably no CB disk is obviously retrograde, ${ }^{14}$ presumably because the dissipative disk-star encounters that most effectively bind binaries do not involve retrograde motions (Borkovits et al. 2016; Tokovinin 2017; Bate 2018).

The two points in the right panel of Figure 14 at $(e$, $\theta) \simeq\left(0.13,45^{\circ}\right)$ and $\left(0.13,50^{\circ}\right)$ pertain to the disk orbiting the GW Ori hierarchical triple. The orbital eccentricities of the triple, which itself is coplanar, appear too low for the mechanism of Martin \& Lubow (2018) to generate the observed disk inclination. The large $\theta$ might instead be a relic of the original dissipative disk-mediated encounter that presumably formed the multiple system (Bate 2018).

\footnotetext{
$\overline{13}$ By contrast, those polar orbits that fall within the observational uncertainties for HD 142527, SR 24N, and GG Tau A do not evince the predicted nodal orientation.

${ }^{14}$ Technically there are observational ambiguities that permit retrograde solutions for HD 200775, R CrA, and IRS 43.
} 
Alternatively, if the GW Ori disk holds the bulk of the system's angular momentum, Lidov-Kozai oscillations may have shaped the system.

\subsection{Circumbinary Planets}

Our finding that $\mathrm{CB}$ disks around short-period, spectroscopic binaries are nearly coplanar $(\theta<3.0)$ adds to the various lines of evidence that Kepler's CB planets-so far discovered with periods shorter than $\sim 300$ days-are similarly coplanar with their host stars. Armstrong et al. (2014) found that by assuming either a strictly coplanar $\mathrm{CB}$ planet population $\left(\theta=0^{\circ}\right)$ or a nearly coplanar distribution centered around $\theta=5^{\circ}$ (similar to our $p_{\text {low }}$ in Section 3.3), the CB planet occurrence rate is either $7_{-1}^{+5} \%$ or $13_{-2}^{+10} \%$, respectively, for planets with radii $\in[4,10] R_{\oplus}$. Their assumed $\theta$-distributions resemble the one we have measured for CB disks around short-period binaries and deliver occurrence rates consistent with that around single stars for similar planet radii and period ranges ( $\sim 8 \%$; Fressin et al. 2013). The interpretation that the Kepler CB planets have low mutual inclinations is also supported by the Bayesian analysis of Li et al. (2016), who used the effects of orbital stability and finite observing time to break the degeneracy between planet occurrence rate and mutual inclination and concluded that $\mathrm{CB}$ planets must on average have $\theta<3^{\circ}$. The BEPOP RV search for $\mathrm{CB}$ planets around single-lined EBs also claims $\theta \lesssim 10^{\circ}$ (Martin et al. 2019).

An enduring curiosity is that EBs having periods $<7$ days have not been found to host CB planets, despite the fact that most known Kepler EBs (whether planet-hosting or not) have shorter periods and thus higher transit probabilities for correspondingly shorter-period, dynamically stable planets (Armstrong et al. 2014; Li et al. 2016). Lidov-Kozai oscillations driven by a tertiary companion have been invoked to tilt planets out of transit or crash them onto their host stars or be ejected (Martin et al. 2015; Muñoz \& Lai 2015), but as previously noted, the role of Lidov-Kozai in forming compact binaries seems limited at best (Moe \& Kratter 2018; Kounkel et al. 2019). Indeed, the two CB disks in our sample around $P<7$ day binaries, V4046 Sgr and CoRoT 2239, have small mutual inclinations and do not host tertiaries capable of driving significant Lidov-Kozai oscillations (V4046 Sgr hosts a companion orbiting $\sim 12,000$ au outside the disk; Kastner et al. 2011). Two promising alternative explanations for the shortfall of CB planets around the shortest-period binaries are (1) a primordial phase of binary orbit expansion driven by pre-mainsequence tidal evolution that destabilizes planetary orbits (Fleming et al. 2018) and (2) that increased X-ray and EUV flux from tight, tidally locked binaries photoevaporates circumbinary planets to smaller planet radii (Sanz-Forcada et al. 2014), which would remain undetected in the Kepler light curves.

\section{Conclusions}

We have analyzed new ALMA observations of the circumbinary (CB) protoplanetary disk around the doublelined spectroscopic binary (SB2) UZ Tau E, finding individual stellar masses of $M_{\mathrm{Ea}}=0.93 \pm 0.04 M_{\odot}$ and $M_{\mathrm{Eb}}=0.28 \pm$ $0.02 M_{\odot}$ and a sky-projected inclination of the binary similar to that of the disk $\left(i_{\star}=56^{\circ} .1 \pm 5^{\circ} .7, \quad i_{\text {disk }}=56.2 \pm 1.5\right)$. UZ Tau E joins a sample of three other CB disks around shortperiod SB2s, all having $i_{\text {disk }} \simeq i_{\star}$ and binary periods $P<20$ days. Although the striking similarity of sky-referenced disk and binary inclinations in these short-period systems suggests that true disk-binary mutual inclinations $\theta$ are small, technically we cannot calculate $\theta$ definitively because the binaries and their disks have unknown relative nodal orientations. We have circumvented this difficulty by implementing a hierarchical Bayesian analysis that fully leverages the observation that $i_{\text {disk }} \simeq i_{\star}$ to infer, in a statistical sense, that short-period binaries are indeed nearly coplanar with their surrounding disks, with $68 \%$ of systems having $\theta<3^{\circ} .0$.

We have assimilated the above sample into a larger collection of the best-characterized circumbinary protoplanetary and debris disks orbiting binaries with periods ranging up to $\sim 10^{5}$ days. Many of these systems have astrometric measurements of the stellar orbit, which enables their mutual inclinations to be calculated directly. Disk-binary mutual inclinations are found to trend strongly with both binary period $P$ and binary eccentricity $e$ : all $\mathrm{CB}$ disks orbiting binaries with $P<30$ days and/or $e<0.2$ are consistent with being coplanar, while $\mathrm{CB}$ disks orbiting longer period and/or more eccentric binaries exhibit a wide range of mutual inclinations, from coplanar to unambiguously polar (HD 98800B and 99 Her).

These trends are consistent with our current understanding of close binary star formation and gravitational torques exerted between host binaries and dissipative CB disks. Binary stars with semimajor axes $<10$ au are thought to form at larger separations and be possibly initially unbound; subsequent dissipative disk-binary gravitational interactions reduce the total system energy and bind/harden the orbit. During these early times, circumbinary disk gas, predominantly prograde, may be initially variously inclined with respect to the binary (Bate 2014, 2018). Those binaries on circular orbits-which the shortest-period binaries tend to be as a result of tidal friction - may drive their CB disks into coplanar alignment, assuming initially modest mutual inclinations (Foucart \& Lai 2013, 2014). Short-period binaries may correlate with initially small disk misalignments insofar as orbital migration driven by $\mathrm{CB}$ disks is more effective at small $\theta$. Eccentric binaries, on the other hand, can induce large disk misalignments. If the initial $\theta$ exceeds a minimum threshold (whose value decreases as the binary eccentricity increases; for $e=0.8$ the minimum $\theta$ is about $30^{\circ}$ ), then $\theta$ can be driven to its equilibrium value of $\approx 90^{\circ}$ (Martin \& Lubow 2017, 2018; Lubow \& Martin 2018; Zanazzi \& Lai 2018). Even if the initial $\theta$ falls short of the critical value, the nonaxisymmetric potential of the eccentric binary can secularly force the CB disk to change its inclination by tens of degrees (Martin \& Lubow 2018; Vinson \& Chiang 2018).

Our finding that $\mathrm{CB}$ disks around short-period binaries are nearly coplanar with their stellar hosts implies (at face value) that planets spawned by such CB disks should be similarly coplanar. We thus add to the growing evidence that Kepler CB planets, orbiting binaries with $P<40$ days, have small mutual inclinations, and that by extension the $\mathrm{CB}$ planet occurrence rate (in this parameter space) is similar to that for single stars (Armstrong et al. 2014; Li et al. 2016; Martin et al. 2019). Beyond $P>40$ days, however, the existence of both aligned and misaligned $\mathrm{CB}$ disks leads us to expect that $\mathrm{CB}$ planets around long-period, eccentric binaries will be discovered to have a correspondingly broad distribution of mutual 
inclinations, with a possible concentration of systems having reached their evolutionary endpoint at $\theta \approx 90^{\circ}$.

I.C. thanks Rob De Rosa, Eric Nielsen, and Lea Hirsch for many helpful discussions about astrometric orbit conventions; Gaspard Duchêne for discussions related to stellar multiplicity; Dan Foreman-Mackey for discussions about gradient-based inference frameworks; David Martin and Daniel Fabrycky for discussions about circumbinary planets; J. J. Zanazzi and Dong Lai for discussions about polar alignment mechanisms; Maxwell Moe for discussions about stellar multiplicity and Lidov-Kozai oscillations; and Jiaqing Bi for discussions about GW Ori and Barinov M.V. for corrections to source names. I.C. and E.C. thank Steve Lubow for discussions. I.C. was supported by NASA through the NASA Hubble Fellowship grant HST-HF2-51405.001-A awarded by the Space Telescope Science Institute, which is operated by the Association of Universities for Research in Astronomy, Inc., for NASA, under contract NAS5-26555. This paper makes use of the following ALMA data: ADS/JAO.ALMA\#2015.1.00690.S. ALMA is a partnership of ESO (representing its member states), NSF (USA) and NINS (Japan), together with NRC (Canada), MOST and ASIAA (Taiwan), and KASI (Republic of Korea), in cooperation with the Republic of Chile. The Joint ALMA Observatory is operated by ESO, AUI/NRAO, and NAOJ. The National Radio Astronomy Observatory is a facility of the National Science Foundation operated under cooperative agreement by Associated Universities, Inc. This research has made use of the NASA/IPAC Extragalactic Database (NED), which is operated by the Jet Propulsion Laboratory, California Institute of Technology, under contract with the National Aeronautics and Space Administration. This research has made use of the SIMBAD database, operated at CDS, Strasbourg, France. This research has made use of NASA's Astrophysics Data System Bibliographic Services.

Software: CASA (v4.4; McMullin et al. 2007), DiskJockey (Czekala et al. 2015, 2019), RADMC-3D (Dullemond 2012), emcee (Foreman-Mackey et al. 2013), Astropy (Astropy Collaboration et al. 2013), PyMC3 (Salvatier et al. 2016), Theano (Theano Development Team 2016), Daft (PGM).

\section{Appendix}

Figures 15 and 16 show the data, model, and residual channel maps for the ${ }^{12} \mathrm{CO}$ and ${ }^{13} \mathrm{CO}$ transitions. 


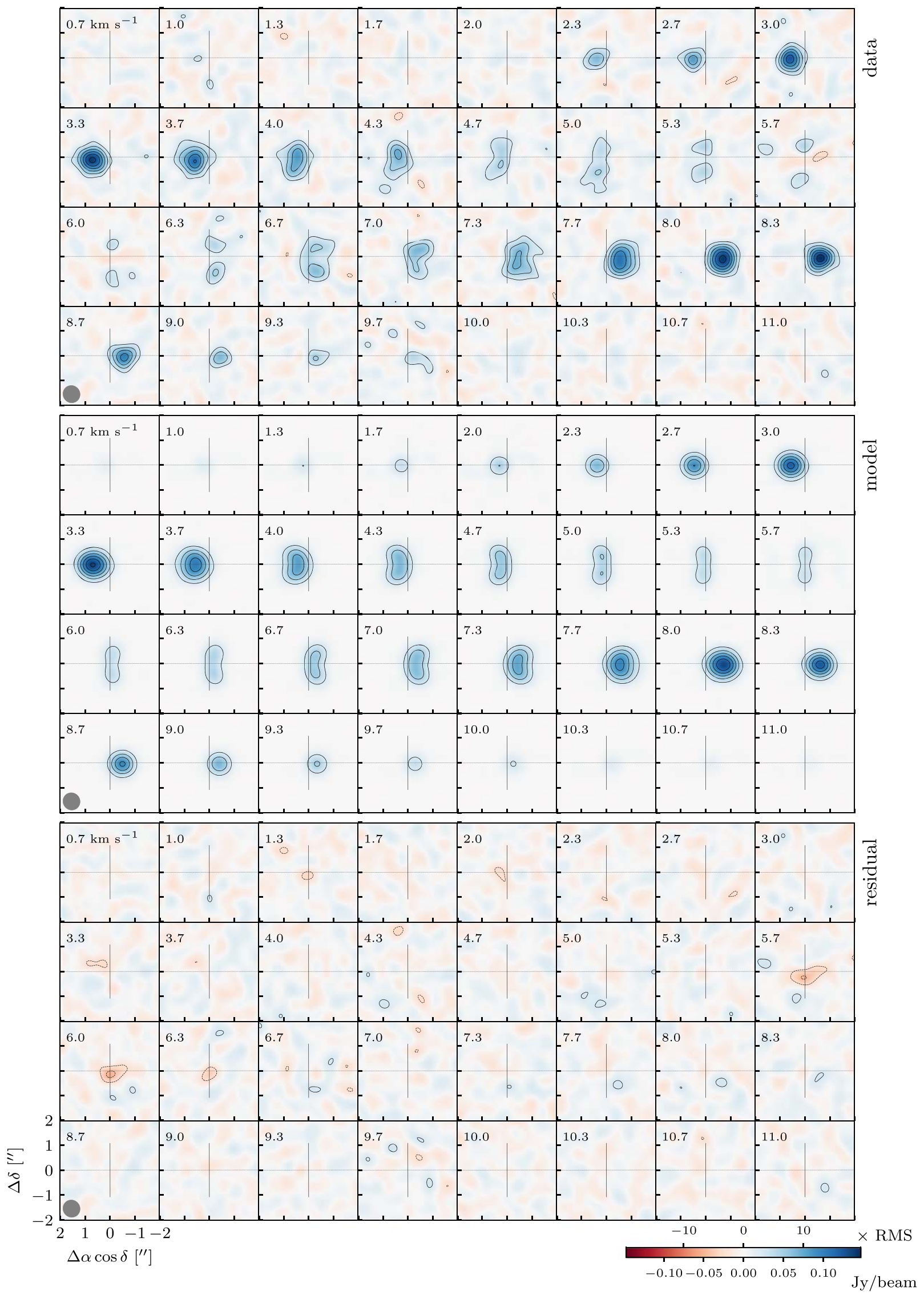

Figure 15. ${ }^{13} \mathrm{CO}$ channel maps centered on UZ Tau E. All velocities are in the LSRK frame. Contours are in multiples of three times the rms. Channels in the range $4.0 \mathrm{~km} \mathrm{~s}^{-1} \leqslant v_{\text {LSRK }} \leqslant 7.5 \mathrm{~km} \mathrm{~s}^{-1}$ were excluded from the fit to avoid cloud contamination. 


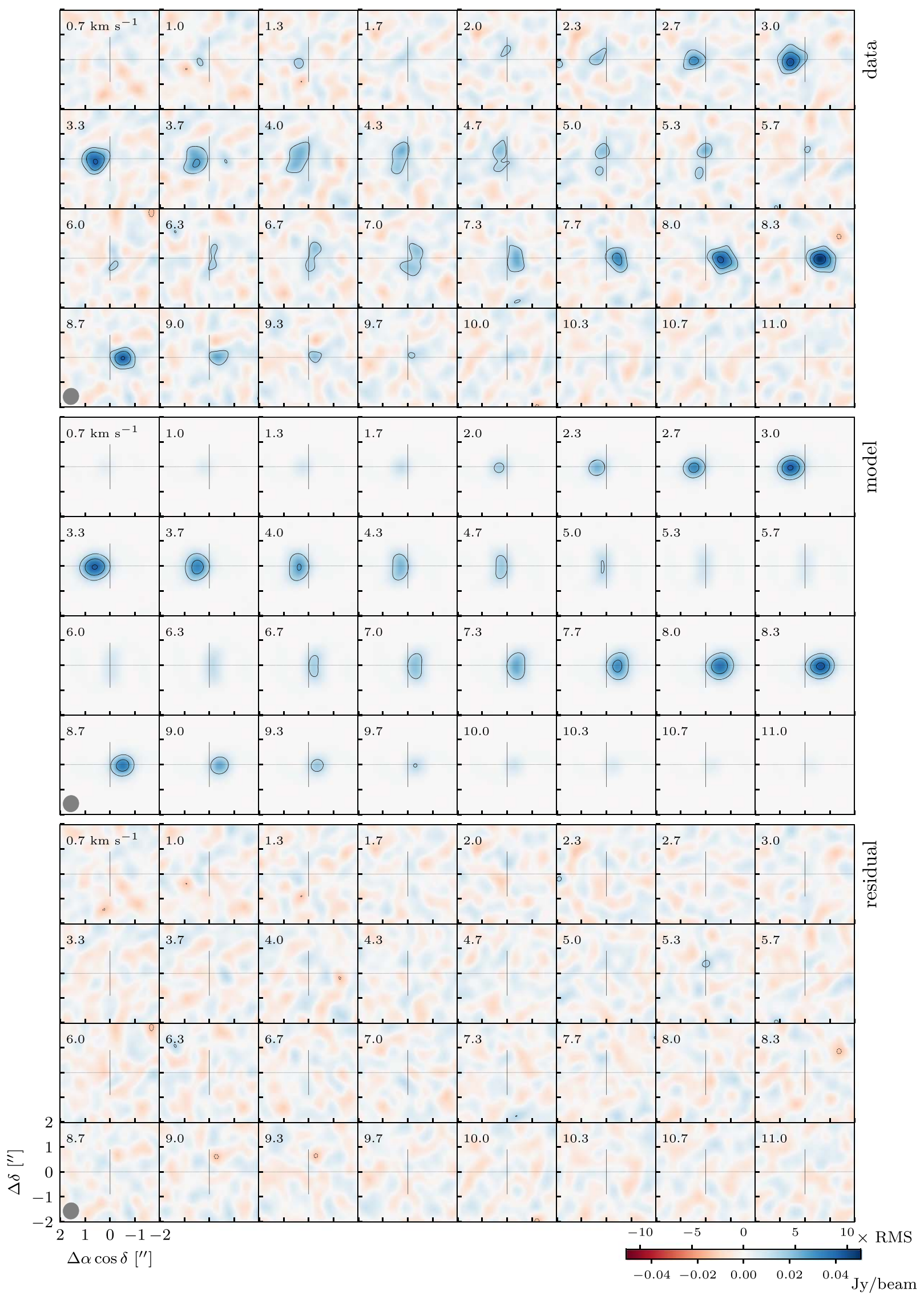

Figure 16. $\mathrm{C}^{18} \mathrm{O}$ channel maps centered on UZ Tau E. All velocities are in the LSRK frame. Contours are in multiples of three times the rms. Channels in the range $4.0 \mathrm{~km} \mathrm{~s}^{-1} \leqslant v_{\text {LSRK }} \leqslant 7.5 \mathrm{~km} \mathrm{~s}^{-1}$ were excluded from the fit to avoid cloud contamination. 


\section{ORCID iDs}

Ian Czekala (1) https://orcid.org/0000-0002-1483-8811 Eugene Chiang (i) https://orcid.org/0000-0002-6246-2310 Sean M. Andrews (iD https://orcid.org/0000-0003-2253-2270 Eric L. N. Jensen (i) https://orcid.org/0000-0002-4625-7333 Guillermo Torres (iD https://orcid.org/0000-0002-5286-0251 David J. Wilner (i) https://orcid.org/0000-0003-1526-7587 Keivan G. Stassun (iD https://orcid.org/0000-0002-3481-9052 Bruce Macintosh (iD https://orcid.org/0000-0003-1212-7538

\section{References}

Alencar, S. H. P., Melo, C. H. F., Dullemond, C. P., et al. 2003, A\&A, 409, 1037

Andrews, S. M., Chandler, C. J., Isella, A., et al. 2014, ApJ, 787, 148

Andrews, S. M., Czekala, I., Wilner, D. J., et al. 2010, ApJ, 710, 462

Andrews, S. M., \& Williams, J. P. 2005, ApJ, 631, 1134

Anthonioz, F., Ménard, F., Pinte, C., et al. 2015, A\&A, 574, A41

Armstrong, D. J., Osborn, H. P., Brown, D. J. A., et al. 2014, MNRAS, 444, 1873

Aronow, R. A., Herbst, W., Hughes, A. M., Wilner, D. J., \& Winn, J. N. 2018 , AJ, 155,47

Artymowicz, P., \& Lubow, S. H. 1996, ApJL, 467, L77

Asensio-Torres, R., Janson, M., Bonavita, M., et al. 2018, A\&A, 619, A43

Astropy Collaboration, Robitaille, T. P., Tollerud, E. J., et al. 2013, A\&A, 558, A33

Bailer-Jones, C. A. L., Rybizki, J., Fouesneau, M., Mantelet, G., \& Andrae, R. 2018, AJ, 156, 58

Bailey, V., Meshkat, T., Reiter, M., et al. 2014, ApJL, 780, L4

Barenfeld, S. A., Carpenter, J. M., Ricci, L., \& Isella, A. 2016, ApJ, 827, 142

Barsony, M., Koresko, C., \& Matthews, K. 2003, ApJ, 591, 1064

Bate, M. R. 2012, MNRAS, 419, 3115

Bate, M. R. 2014, MNRAS, 442, 285

Bate, M. R. 2018, MNRAS, 475, 5618

Bate, M. R. 2019, MNRAS, 484, 2341

Bate, M. R., Bonnell, I. A., \& Bromm, V. 2002, MNRAS, 336, 705

Benisty, M., Perraut, K., Mourard, D., et al. 2013, A\&A, 555, A113

Bennett, D. P., Rhie, S. H., Udalski, A., et al. 2016, AJ, 152, 125

Betancourt, M. 2017, arXiv:1701.02434

Betancourt, M. J., \& Girolami, M. 2013, arXiv:1312.0906

Biller, B., Lacour, S., Juhász, A., et al. 2012, ApJL, 753, L38

Boden, A. F., Sargent, A. I., Akeson, R. L., et al. 2005, ApJ, 635, 442

Boehler, Y., Weaver, E., Isella, A., et al. 2017, ApJ, 840, 60

Böhm, T., Catala, C., Balona, L., \& Carter, B. 2004, A\&A, 427, 907

Borkovits, T., Hajdu, T., Sztakovics, J., et al. 2016, MNRAS, 455, 4136

Brinch, C., Jørgensen, J. K., Hogerheijde, M. R., Nelson, R. P., \& Gressel, O. 2016, ApJL, 830, L16

Cazzoletti, P., Ricci, L., Birnstiel, T., \& Lodato, G. 2017, A\&A, 599, A102

Chiang, E. I., \& Murray-Clay, R. A. 2004, ApJ, 607, 913

Cieza, L. A., Schreiber, M. R., Romero, G. A., et al. 2010, ApJ, 712, 925

Claudi, R., Maire, A. L., Mesa, D., et al. 2019, A\&A, 622, A96

Close, L. M., Dutrey, A., Roddier, F., et al. 1998, ApJ, 499, 883

Comerón, F., Reipurth, B., Yen, H.-W., \& Connelley, M. S. 2018, A\&A, 612, A73

Correia, S., Zinnecker, H., Ratzka, T., \& Sterzik, M. F. 2006, A\&A, 459, 909

Cox, E. G., Harris, R. J., Looney, L. W., et al. 2017, ApJ, 851, 83

Czekala, I., Andrews, S. M., Jensen, E. L. N., et al. 2015, ApJ, 806, 154

Czekala, I., Andrews, S. M., Torres, G., et al. 2016, ApJ, 818, 156

Czekala, I., Andrews, S. M., Torres, G., et al. 2017, ApJ, 851, 132

Czekala, I., Jensen, E., \& Huang, J. 2019, iancze/DiskJockey: Upgrades to Julia v1.0, Zenodo, doi:10.5281/zenodo. 3235028

D’Alessio, P., Hartmann, L., Calvet, N., et al. 2005, ApJ, 621, 461

De Rosa, R. J., \& Kalas, P. 2019, AJ, 157, 125

Di Folco, E., Dutrey, A., Le Bouquin, J. B., et al. 2014, A\&A, 565, L2

Dong, R., Fung, J., \& Chiang, E. 2016, ApJ, 826, 75

Doppmann, G. W., Najita, J. R., \& Carr, J. S. 2008, ApJ, 685, 298

Doyle, L. R., Carter, J. A., Fabrycky, D. C., et al. 2011, Sci, 333, 1602

Dullemond, C. P. 2012, RADMC-3D: A Multi-purpose Radiative Transfer Tool, Astrophysics Source Code, ascl:1202.015

Dunham, M. M., Offner, S. S. R., Pineda, J. E., et al. 2016, ApJ, 823, 160

Dutrey, A., Di Folco, E., Beck, T., \& Guilloteau, S. 2016, A\&ARv, 24, 5

Dvorak, R. 1982, OAWMN, 191, 423
Eisner, J. A., Hillenbrand, L. A., White, R. J., Akeson, R. L., \& Sargent, A. I. 2005, ApJ, 623, 952

Espaillat, C., Furlan, E., D’Alessio, P., et al. 2011, ApJ, 728, 49

Farago, F., \& Laskar, J. 2010, MNRAS, 401, 1189

Fernández-López, M., Zapata, L. A., \& Gabbasov, R. 2017, ApJ, 845, 10

Fleming, D. P., Barnes, R., Graham, D. E., Luger, R., \& Quinn, T. R. 2018, ApJ, 858, 86

Foreman-Mackey, D., Hogg, D. W., Lang, D., \& Goodman, J. 2013, PASP, 125,306

Foucart, F., \& Lai, D. 2013, ApJ, 764, 106

Foucart, F., \& Lai, D. 2014, MNRAS, 445, 1731

Fressin, F., Torres, G., Charbonneau, D., et al. 2013, ApJ, 766, 81

Gaia Collaboration, Brown, A. G. A., Vallenari, A., et al. 2018, A\&A, 616, A1

Garcia, P. J. V., Benisty, M., Dougados, C., et al. 2013, MNRAS, 430, 1839

Gelman, A., Carlin, J. B., Stern, H. S., et al. 2014, Bayesian Data Analysis, Vol. 2 (Boca Raton, FL: CRC Press)

Ghez, A. M., Neugebauer, G., \& Matthews, K. 1993, AJ, 106, 2005

Gillen, E., Aigrain, S., McQuillan, A., et al. 2014, A\&A, 562, A50

Gillen, E., Aigrain, S., Terquem, C., et al. 2017, A\&A, 599, A27

Ginski, C., Benisty, M., van Holstein, R. G., et al. 2018, A\&A, 616, A79

Goldsmith, P. F., Heyer, M., Narayanan, G., et al. 2008, ApJ, 680, 428

Goodman, J., \& Weare, J. 2010, Communications in Applied Mathematics and Computational Science, 5, 65

Guenther, E. W., Esposito, M., Mundt, R., et al. 2007, A\&A, 467, 1147

Guilloteau, S., Di Folco, E., Dutrey, A., et al. 2013, A\&A, 549, A92

Guilloteau, S., Dutrey, A., Piétu, V., \& Boehler, Y. 2011, A\&A, 529, A105

Hamilton, C. M., Herbst, W., Mundt, R., Bailer-Jones, C. A. L., \& Johns-Krull, C. M. 2003, ApJL, 591, L45

Hamilton, C. M., Herbst, W., Shih, C., \& Ferro, A. J. 2001, ApJL, 554, L201

Hamilton, C. M., Herbst, W., Vrba, F. J., et al. 2005, AJ, 130, 1896

Harris, R. J., Andrews, S. M., Wilner, D. J., \& Kraus, A. L. 2012, ApJ, 751,115

Harris, R. J., Cox, E. G., Looney, L. W., et al. 2018, ApJ, 861, 91

Hartmann, L., Hewett, R., Stahler, S., \& Mathieu, R. D. 1986, ApJ, 309, 275

Herbst, W., Hamilton, C. M., Vrba, F. J., et al. 2002, PASP, 114, 1167

Hioki, T., Itoh, Y., Oasa, Y., et al. 2007, AJ, 134, 880

Hoffman, M. D., \& Gelman, A. 2014, Journal of Machine Learning Research, 15,1593

Hogg, D. W., Myers, A. D., \& Bovy, J. 2010, ApJ, 725, 2166

Ireland, M. J., \& Kraus, A. L. 2008, ApJL, 678, L59

Janson, M., Thalmann, C., Boccaletti, A., et al. 2016, ApJL, 816, L1

Jensen, E. L. N., Dhital, S., Stassun, K. G., et al. 2007, AJ, 134, 241

Jensen, E. L. N., Koerner, D. W., \& Mathieu, R. D. 1996a, AJ, 111, 2431

Jensen, E. L. N., Mathieu, R. D., \& Fuller, G. A. 1996b, ApJ, 458, 312

Johnson, J. A., \& Winn, J. N. 2004, AJ, 127, 2344

Kalas, P. G., Rajan, A., Wang, J. J., et al. 2015, ApJ, 814, 32

Kastner, J. H. 2018, RNAAS, 2, 137

Kastner, J. H., Qi, C., Dickson-Vandervelde, D. A., et al. 2018, ApJ, 863, 106

Kastner, J. H., Sacco, G. G., Montez, R., et al. 2011, ApJL, 740, L17

Kellogg, K., Prato, L., Torres, G., et al. 2017, ApJ, 844, 168

Kennedy, G. M. 2015, MNRAS, 447, L75

Kennedy, G. M., Matrà, L., Facchini, S., et al. 2019, NatAs, 3, 230

Kennedy, G. M., Wyatt, M. C., Sibthorpe, B., et al. 2012a, MNRAS, 421, 2264

Kennedy, G. M., Wyatt, M. C., Sibthorpe, B., et al. 2012b, MNRAS, 426, 2115

Kohn, S. A., Shkolnik, E. L., Weinberger, A. J., Carlberg, J. K., \& Llama, J. 2016, ApJ, 820, 2

Kounkel, M., Covey, K., Moe, M., et al. 2019, AJ, 157, 196

Kratter, K. M., Matzner, C. D., \& Krumholz, M. R. 2008, ApJ, 681, 375

Kraus, A. L., Ireland, M. J., Huber, D., Mann, A. W., \& Dupuy, T. J. 2016, AJ, 152,8

Kraus, A. L., Ireland, M. J., Martinache, F., \& Hillenbrand, L. A. 2011, ApJ, 731,8

Kraus, S., Hofmann, K. H., Malbet, F., et al. 2009, A\&A, 508, 787

Kraus, S., Kluska, J., Kreplin, A., et al. 2017, Msngr, 170, 45

Kurtovic, N. T., Pérez, L. M., Benisty, M., et al. 2018, ApJL, 869, L44

Lacour, S., Biller, B., Cheetham, A., et al. 2016, A\&A, 590, A90

Larson, R. B. 1969, MNRAS, 145, 271

Lee, C. W., Martin, E. L., \& Mathieu, R. D. 1994, AJ, 108, 1445

Li, G., Holman, M. J., \& Tao, M. 2016, ApJ, 831, 96

Lindegren, L., Hernández, J., Bombrun, A., et al. 2018, A\&A, 616, A2

Lodato, G., \& Facchini, S. 2013, MNRAS, 433, 2157

Long, F., Pinilla, P., Herczeg, G. J., et al. 2018, ApJ, 869, 17

Loredo, T. J. 2013, Astrostatistical Challenges for the New Astronomy, ed. J. M. Hilbe (New York: Springer), 15

Lubow, S. H., \& Martin, R. G. 2018, MNRAS, 473, 3733 
Mackay, D. J. C. 2003, Information Theory, Inference and Learning Algorithms (Cambridge: Cambridge Univ. Press)

Martin, D. V., Mazeh, T., \& Fabrycky, D. C. 2015, MNRAS, 453, 3554

Martin, D. V., \& Triaud, A. H. M. J. 2014, A\&A, 570, A91

Martin, D. V., Triaud, A. H. M. J., Udry, S., et al. 2019, A\&A, 624, A68

Martín, E. L., Magazzù, A., Delfosse, X., \& Mathieu, R. D. 2005, A\&A, 429, 939

Martin, R. G., \& Lubow, S. H. 2017, ApJL, 835, L28

Martin, R. G., \& Lubow, S. H. 2018, MNRAS, 479, 1297

Martin, R. G., \& Lubow, S. H. 2019, arXiv:1904.11631

Mathieu, R. D. 1994, ARA\&A, 32, 465

Mathieu, R. D., Martin, E. L., \& Maguzzu, A. 1996, BAAS, 28, 920

Mathieu, R. D., Walter, F. M., \& Myers, P. C. 1989, AJ, 98, 987

Matthews, B. C., Sibthorpe, B., Kennedy, G., et al. 2010, A\&A, 518, L135

McMullin, J. P., Waters, B., Schiebel, D., Young, W., \& Golap, K. 2007, in ASP Conf. Ser. 376, Astronomical Data Analysis Software and Systems XVI, ed. R. A. Shaw, F. Hill, \& D. J. Bell (San Francisco, CA: ASP), 127 Melo, C. H. F. 2003, A\&A, 410, 269

Menard, F., Monin, J.-L., Angelucci, F., \& Rouan, D. 1993, ApJL, 414, L117

Mesa, D., Bonnefoy, M., Gratton, R., et al. 2019, A\&A, 624, A4

Moe, M., \& Kratter, K. M. 2018, ApJ, 854, 44

Monnier, J. D., Berger, J. P., Millan-Gabet, R., et al. 2006, ApJ, 647, 444

Monnier, J. D., Harries, T. J., Bae, J., et al. 2019, ApJ, 872, 122

Monnier, J. D., Tannirkulam, A., Tuthill, P. G., et al. 2008, ApJL, 681, L97

Muñoz, D. J., \& Lai, D. 2015, PNAS, 112, 9264

Muñoz, D. J., \& Perets, H. B. 2018, AJ, 156, 253

Murray, C. D., \& Correia, A. C. M. 2010, in Keplerian Orbits and Dynamics of Exoplanets, ed. S. Seager (Tucson, AZ: Univ. Arizona Press), 15

Nagel, E., D’Alessio, P., Calvet, N., et al. 2010, ApJ, 708, 38

Naoz, S., Li, G., Zanardi, M., de Elía, G. C., \& Di Sisto, R. P. 2017, AJ, 154,18

Narayanan, G., Heyer, M. H., Brunt, C., et al. 2008, ApJS, 177, 341

Offner, S. S. R., Kratter, K. M., Matzner, C. D., Krumholz, M. R., \& Klein, R. I. 2010, ApJ, 725, 1485

Okamoto, Y. K., Kataza, H., Honda, M., et al. 2009, ApJ, 706, 665

Orellana, M., Cieza, L. A., Schreiber, M. R., et al. 2012, A\&A, 539, A41

Orosz, J. A., Welsh, W. F., Carter, J. A., et al. 2012, ApJ, 758, 87

Plavchan, P., Gee, A. H., Stapelfeldt, K., \& Becker, A. 2008, ApJL, 684, L37

Plavchan, P., Güth, T., Laohakunakorn, N., \& Parks, J. R. 2013, A\&A, 554, A110

Pourbaix, D. 1998, A\&AS, 131, 377

Prato, L., Simon, M., Mazeh, T., Zucker, S., \& McLean, I. S. 2002, ApJL, 579, L99

Price, D. J., Cuello, N., Pinte, C., et al. 2018, MNRAS, 477, 1270

Raghavan, D., McAlister, H. A., Henry, T. J., et al. 2010, ApJS, 190, 1

Ratzka, T., Köhler, R., \& Leinert, C. 2005, A\&A, 437, 611
Ribas, Á., Macías, E., Espaillat, C. C., \& Duchêne, G. 2018, ApJ, 865, 77 Rodriguez, D. R., Duchêne, G., Tom, H., et al. 2015, MNRAS, 449, 3160 Rosenfeld, K. A., Andrews, S. M., Hughes, A. M., Wilner, D. J., \& Qi, C. 2013, ApJ, 774, 16

Rosenfeld, K. A., Andrews, S. M., Wilner, D. J., \& Stempels, H. C. 2012, ApJ, 759, 119

Ruíz-Rodríguez, D., Cieza, L. A., Williams, J. P., et al. 2018, MNRAS, 478, 3674

Ruíz-Rodríguez, D., Ireland, M., Cieza, L., \& Kraus, A. 2016, MNRAS, 463, 3829

Salvatier, J., Wiecki, T. V., \& Fonnesbeck, C. 2016, PeerJ Computer Science, 2, e55

Sanz-Forcada, J., Desidera, S., \& Micela, G. 2014, A\&A, 570, A50

Schaefer, G. H., Prato, L., \& Simon, M. 2018, AJ, 155, 109

Schwamb, M. E., Orosz, J. A., Carter, J. A., et al. 2013, ApJ, 768, 127

Sharma, S. 2017, ARA\&A, 55, 213

Sheehan, P. D., \& Eisner, J. A. 2014, ApJ, 791, 19

Simon, M., Dutrey, A., \& Guilloteau, S. 2000, ApJ, 545, 1034

Smith, K. W., Balega, Y. Y., Duschl, W. J., et al. 2005, A\&A, 431, 307

Stempels, H. C., \& Gahm, G. F. 2004, A\&A, 421, 1159

Takakuwa, S., Saigo, K., Matsumoto, T., et al. 2017, ApJ, 837, 86

Tang, Y.-W., Dutrey, A., Guilloteau, S., et al. 2014, ApJ, 793, 10

Tang, Y.-W., Dutrey, A., Guilloteau, S., et al. 2016, ApJ, 820, 19

Teague, R., \& Foreman-Mackey, D. 2018, RNAAS, 2, 173

Terquem, C., Sørensen-Clark, P. M., \& Bouvier, J. 2015, MNRAS, 454, 3472

Theano Development Team 2016, arXiv:1605.02688

Tobin, J. J., Kratter, K. M., Persson, M. V., et al. 2016, Natur, 538, 483

Tokovinin, A. 2017, ApJ, 844, 103

Tokovinin, A., Thomas, S., Sterzik, M., \& Udry, S. 2006, A\&A, 450, 681

Torres, G. 1995, PASP, 107, 524

Torres, G. 2004, AJ, 127, 1187

Tripathi, A., Andrews, S. M., Birnstiel, T., et al. 2018, ApJ, 861, 64

Udry, S., Mayor, M., Naef, D., et al. 2002, A\&A, 390, 267

van de Kamp, P. 1981, in Stellar Paths: Photographic Astrometry with Longfocus Instruments, ed. P. van de Kamp (Dordrecht: Reidel)

Verrier, P. E., \& Evans, N. W. 2009, MNRAS, 394, 1721

Vinson, B. R., \& Chiang, E. 2018, MNRAS, 474, 4855

Wang, J., Fischer, D. A., Xie, J.-W., \& Ciardi, D. R. 2014, ApJ, 791, 111

Welsh, W. F., Orosz, J. A., Carter, J. A., et al. 2012, Natur, 481, 475

Winn, J. N., \& Fabrycky, D. C. 2015, ARA\&A, 53, 409

Winn, J. N., Garnavich, P. M., Stanek, K. Z., \& Sasselov, D. D. 2003, ApJL, 593, L121

Winn, J. N., Hamilton, C. M., Herbst, W. J., et al. 2006, ApJ, 644, 510

Wolfgang, A., \& Lopez, E. 2015, ApJ, 806, 183

Zanazzi, J. J., \& Lai, D. 2018, MNRAS, 473, 603 